\title{
AN IMPROVED UPPER BOUND FOR BOOTSTRAP PERCOLATION IN ALL DIMENSIONS
}

\author{
ANDREW J. UZZELL
}

\begin{abstract}
In $r$-neighbor bootstrap percolation on the vertex set of a graph $G$, a set $A$ of initially infected vertices spreads by infecting, at each time step, all uninfected vertices with at least $r$ previously infected neighbors. When the elements of $A$ are chosen independently with some probability $p$, it is natural to study the critical probability $p_{c}(G, r)$ at which it becomes likely that all of $V(G)$ will eventually become infected. Improving a result of Balogh, Bollobás, and Morris, we give a bound on the second term in the expansion of the critical probability when $G=[n]^{d}$ and $d \geq r \geq 2$. We show that for all $d \geq r \geq 2$ there exists a constant $c_{d, r}>0$ such that if $n$ is sufficiently large, then
\end{abstract}

$$
p_{c}\left([n]^{d}, r\right) \leq\left(\frac{\lambda(d, r)}{\log _{(r-1)}(n)}-\frac{c_{d, r}}{\left(\log _{(r-1)}(n)\right)^{3 / 2}}\right)^{d-r+1},
$$

where $\lambda(d, r)$ is an exact constant and $\log _{(k)}(n)$ denotes the $k$-times iterated natural logarithm of $n$.

\section{INTRODUCTION}

Bootstrap percolation on the vertex set of a graph is a cellular automaton in which vertices have two possible states, "infected" and "uninfected". Let $r \in \mathbb{N}$ and let $G$ be a graph. In $r$-neighbor bootstrap percolation, a set $A \subseteq$ $V(G)$ is infected at time 0 . At each subsequent time step, all infected vertices remain infected and all uninfected vertices with at least $r$ infected neighbors become infected. In symbols, letting $A_{0}=A$, we have

$$
A_{t+1}=A_{t} \cup\left\{v:\left|N(v) \cap A_{t}\right| \geq r\right\}
$$

for all $t \geq 0$. Define the closure of $A$ to be $[A]:=\bigcup_{t=0}^{\infty} A_{t}$, the set of vertices that eventually become infected. If $[A]=V(G)$, we say that $A$ percolates $G$, or simply that $G$ percolates.

Bootstrap percolation was introduced by Chalupa, Leath, and Reich [12] in connection with the Blume-Capel model of ferromagnetism.

Here, as often in the literature, elements of $A$ are chosen independently with some probability $p$. Given $p \in[0,1]$, we define $P(G, r, p)$ to be the probability that $A$ percolates $G$ under the $r$-neighbor rule if the elements of $A$ are chosen in this way. We define $p_{\alpha}(G, r)=\inf \{p: P(G, r, p) \geq \alpha\}$

This work was done while the author was at the University of Memphis. 
and let

$$
p_{c}:=p_{c}(G, r):=p_{1 / 2}(G, r)
$$

denote the critical probability.

Van Enter [25] and Schonmann [24] showed that for all $d \geq 2, p_{c}\left(\mathbb{Z}^{d}, r\right)=0$ when $r \leq d$ and $p_{c}\left(\mathbb{Z}^{d}, r\right)=1$ when $r \geq d+1$. Since then, much work has focused on $p_{c}\left([n]^{d}, r\right)$ (where $[n]=\{1, \ldots, n\}$ ) for $2 \leq r \leq d$. In this case, the critical probability of percolation displays a sharp threshold. That is, for all $\varepsilon>0$ and $n$ sufficiently large, if $p>(1+\varepsilon) p_{c}$, then $P\left([n]^{d}, r, p\right)>1-\varepsilon$, and if $p<(1-\varepsilon) p_{c}$, then $P\left([n]^{d}, r, p\right)<\varepsilon$. Aizenman and Lebowitz [2] determined that for all $d \geq 2, p_{c}\left([n]^{d}, 2\right)=\Theta(1 / \log n)^{d-1}$. (Later, Balogh and Pete [6] independently proved this result for $d=2$.) Cerf and Cirillo [10] and Cerf and Manzo [11] showed that for all $d \geq r \geq 2$, $p_{c}\left([n]^{d}, r\right)=\Theta\left(1 / \log _{(r-1)}(n)\right)^{d-r+1}$, where $\log _{(k)}(n)$ denotes the $k$-times iterated natural logarithm of $n$, so that $\log _{(k)}(n)=\log \left(\log _{(k-1)}(n)\right)$ and $\log _{(1)}(n)=\log n$.

The next breakthrough in the field was due to Holroyd [21], who proved a sharp threshold result for bootstrap percolation on the two-dimensional grid.

Theorem 1.1. As $n \rightarrow \infty$,

$$
p_{c}\left([n]^{2}, 2\right)=\frac{\pi^{2}}{18 \log n}+o\left(\frac{1}{\log n}\right) .
$$

Later, Gravner and Holroyd [17], Gravner, Holroyd, and Morris [18], and Hartarsky and Morris [20] sharpened Holroyd's result. Collectively, they proved the following.

Theorem 1.2. There exist constants $C \geq c>0$ such that

$$
\frac{\pi^{2}}{18 \log n}-\frac{C}{(\log n)^{3 / 2}} \leq p_{c}\left([n]^{2}, 2\right) \leq \frac{\pi^{2}}{18 \log n}-\frac{c}{(\log n)^{3 / 2}}
$$

for all $n$ sufficiently large.

Turning to higher dimensions, Balogh, Bollobás, and Morris [5] proved a sharp threshold result for $p_{c}\left([n]^{3}, 3\right)$ and proved an upper bound on $p_{c}\left([n]^{d}, r\right)$ for all constant $d \geq r \geq 2$. Later, Balogh, Bollobás, Duminil-Copin, and Morris [4] proved the corresponding lower bound and so established the sharp threshold result for all constant $d \geq r \geq 2$. These results are substantially more difficult than the proof of Theorem 1.1.

Before we state the results of $[4,5]$, we need to introduce more notation. Given $k \geq 1$, define the function $\beta_{k}:(0,1) \rightarrow(0,1)$ by

$$
\beta_{k}(u)=\frac{1}{2}\left(1-(1-u)^{k}+\sqrt{1+(4 u-2)(1-u)^{k}+(1-u)^{2 k}}\right)
$$

and let

$$
g_{k}(z)=-\log \left(\beta_{k}\left(1-e^{-z}\right)\right) .
$$


For $d \geq r \geq 2$, define

$$
\lambda(d, r)=\int_{0}^{\infty} g_{r-1}\left(z^{d-r+1}\right) d z .
$$

Holroyd [21] showed that $\lambda(2,2)=\pi^{2} / 18$. At present, $(2,2)$ is the only ordered pair $(d, r)$ for which an exact expression for $\lambda(d, r)$ is known. However, it is shown in [5] that $\lambda(d, r)<\infty$ for all $d \geq r \geq 2$.

Here is the sharp threshold result of Balogh, Bollobás, Duminil-Copin, and Morris [4, 5].

Theorem 1.3. Let $d \geq r \geq 2$. With $\lambda(d, r)$ as defined in (1.3),

$$
p_{c}\left([n]^{d}, r\right)=\left(\frac{\lambda(d, r)+o(1)}{\log _{(r-1)}(n)}\right)^{d-r+1} .
$$

A number of variations of the bootstrap process described above have been considered. Holroyd [21, 22] proved, for all $d \geq 2$, a sharp threshold result for a modified $d$-neighbor bootstrap rule on $[n]^{d}$ : in order to become infected, a vertex must have at least one infected neighbor in each dimension. Sharp threshold results have also been proved for other update rules on $\mathbb{Z}^{d}$ and $[n]^{d}[8,14,15,13,26]$. Similar but weaker results about the threshold behavior of a very general class of update rules on $\mathbb{Z}^{2}$ were proved in $[3,7,9]$.

Bootstrap percolation has been applied to other fields, especially physics. In particular, there is a strong connection between bootstrap percolation and the Glauber dynamics of the Ising model of ferromagnetism at zero temperature [2, 16, 23]. For other applications in physics, see [1] and the references therein.

In [4], Balogh, Bollobás, Duminil-Copin, and Morris suggested that the techniques of [18] could be used to prove an analogue of Theorem 1.2 for $p_{c}\left([n]^{d}, 2\right)$. We carry this program out in part. We combine the techniques of [17] and [5] to improve the upper bound on $p_{c}\left([n]^{d}, r\right)$ given in Theorem 1.3 for all $d \geq r \geq 2$.

Theorem 1.4. For all $d \geq r \geq 2$, there exists a constant $c_{d, r}>0$ such that

$$
p_{c}\left([n]^{d}, r\right) \leq\left(\frac{\lambda(d, r)}{\log _{(r-1)}(n)}-\frac{c_{d, r}}{\left(\log _{(r-1)}(n)\right)^{3 / 2}}\right)^{d-r+1}
$$

for all $n$ sufficiently large.

We note that when $d=r=2,(1.4)$ reduces to the upper bound in Theorem 1.2, which was proved by Gravner and Holroyd [17].

The rest of the paper is organized as follows. In Section 2, we give an outline of the proof of Theorem 1.4. In Section 3, we introduce additional notation and some preliminary results. In Section 4, we state an important auxiliary result, Theorem 4.1, and also state and prove other auxiliary results. In Section 5, we prove Theorem 4.1 in the case $r=2$. In Section 6, we complete the proof of Theorem 4.1 and use it to deduce Theorem 1.4. Finally, in Section 7, we conjecture an improved lower bound on $p_{c}\left([n]^{d}, r\right)$. 


\section{Outline of the Proof of Theorem 1.4}

Here we will sketch the proof of Theorem 1.4. Our argument builds on a large body of previous work (in particular, [2], [21], [17], [11], and [5]). We hope that discussing the relevant ideas from these papers at some length will serve to make our proof clearer to the reader.

We begin with a few definitions. In the literature of percolation theory, vertices of a graph are often called sites, and we will almost always use this term hereafter. We say that a set $S \subseteq[n]^{d}$ is internally spanned if $[A \cap S]=S$. We say that a set of vertices is empty or unoccupied if it contains no infected sites and occupied otherwise. We say that a sequence of events $E_{1}, \ldots, E_{n}$ has a double gap if some pair of consecutive events $\left(E_{i}, E_{i+1}\right)$ does not occur. Finally, given $r \geq 3$, let $\mathbf{1}^{r-2}$ denote the vector $(1, \ldots, 1) \in \mathbb{R}^{r-2}$ and, for each $i \in[r-2]$, let $e_{i}$ denote the $i$ th standard basis vector.

2.1. Two Dimensions. One might suppose that if $[n]^{d}$ percolates, then the infected set spreads to all parts of the grid in a fairly uniform manner. In [2], Aizenman and Lebowitz showed that in fact, when the infection probability $p$ is on the order of $(1 / \log n)^{d-1}$, whether percolation occurs under the 2neighbor rule is governed by a more local phenomenon: the existence of a fairly small internally spanned set, called a critical droplet. For example, for 2-neighbor percolation in $[n]^{2}$, a natural candidate for a critical droplet is a rectangle whose diameter (in the $L^{\infty}$ norm) is on the order of $\log n$. (A heuristic explanation for this is given in Section 2.2.)

So, in [21], Holroyd proved the upper bound on $p_{c}\left([n]^{2}, 2\right)$ in Theorem 1.1 by estimating the probability that a square $R$ of side length $B: \approx \log n$ is internally spanned in a certain way.

Let $a \ll B$ and let $S$ denote the copy of $[a]^{2}$ in the lower left corner of $R$. If $S$ is fully infected, what conditions imply that the infected set will grow from $S$ to fill $R$ ? If the rows $[a] \times\{a+1\},[a] \times\{a+2\}, \ldots,[a] \times\{B\}$ are all occupied, then these rows will iteratively become infected. If the same holds for the columns $\{i\} \times[a]$, then all of $[B]^{2}$ will become infected.

Holroyd observed that we can get away with asking for a bit less. Note that if either of the rows $[a] \times\{a+1\}$ and $[a] \times\{a+2\}$ contains an infected site, then all sites in the row $[a] \times\{a+1\}$ will become fully infected. (Much the same is true for the columns $\{a+1\} \times[a]$ and $\{a+2\} \times[a]$.) Motivated by this observation, we let $R_{i}$ denote the event that $[i-1] \times\{i\}$ is occupied, let $C_{i}$ denote the event that $\{i\} \times[i-1]$ is occupied, and let $D$ denote the event that the sequences $\left(R_{i}\right)_{i=a+1}^{B+1}$ and $\left(C_{i}\right)_{i=a+1}^{B+1}$ each contain no double gaps. Observe that if $D$ occurs, then the infected set will grow from $S$ to fill $R$. We think of $D$ as "diagonal growth" of the infected set, because the infected set iteratively fills the sets $[t]^{2}$ for $t=a+1, \ldots, B$ (see Figure 1).

As the reader might guess, the probability that $D$ occurs is fairly small. However, it is large enough that if $[n]^{2}$ is partitioned into squares of side length $B$, then with high probability $D$ occurs in some square. Furthermore, 


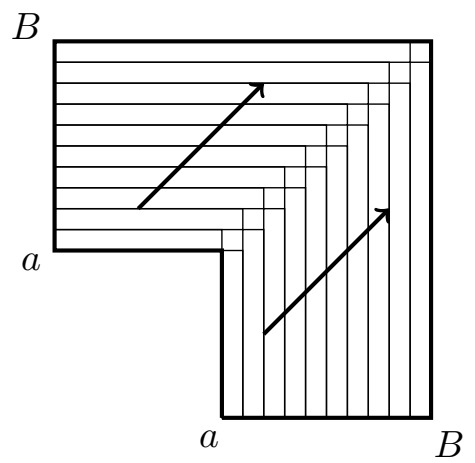

FIgURE 1. If no two consecutive rows and no two consecutive columns are unoccupied, then the infected set will grow diagonally.

if such a square is fully infected, then with high probability the infected set will fill the entire grid.

How might one prove a stronger upper bound on $p_{c}\left([n]^{2}, 2\right)$ ? Instead of considering a single event that implies that the square $R$ is internally spanned, one might consider a set of pairwise disjoint events $E_{1}, \ldots, E_{N}$, for some $N=N(p)$, each of which implies that $R$ is internally spanned. If, for each $i$, we had $\mathbb{P}\left(E_{i}\right) \geq\left(c_{1} p\right)^{1 / p^{2}} \mathbb{P}(D)$, and if $N=\left(c_{2} / p\right)^{1 / p^{2}}$ (where $c_{1}$ and $c_{2}$ are constants such that $\left.c_{1} c_{2}>1\right)$, then we would have

$$
\mathbb{P}\left(\bigvee_{i=1}^{N} E_{i}\right) \geq\left(c_{2} / p\right)^{1 / p^{2}}\left(c_{1} p\right)^{1 / p^{2}} \mathbb{P}(D)=e^{c / p^{2}} \mathbb{P}(D)
$$

where $c:=\log \left(c_{1} c_{2}\right)>0$. It turns out that the factor $e^{c / p^{2}}$ on the right-hand side of (2.1) is enough to make a difference in the value of $p_{c}\left([n]^{2}, 2\right)$ and to prove the upper bound in Theorem 1.2.

Gravner and Holroyd [17] did precisely this. They considered the event that $R$ is internally spanned, but that at some point, a double gap in either $\left(R_{i}\right)$ or $\left(C_{i}\right)$ creates a small "detour" in the diagonal growth of the infected set. Once again, consider the fully infected square $S=[a]^{2}$, and suppose that for some $b>a$, the rows $[b-1] \times\{a+1\}$ and $[b-1] \times\{a+2\}$ are both empty. Clearly, this double gap blocks the infected set from growing vertically. However, if the columns to the right of $S$ contain no double gaps until at least column $b+1$, then the infected set can grow horizontally until it fills the rectangle $[b] \times[a]$. If the infected set eventually encounters an infected site above $[b] \times[a]$ (for example, $(b, a+2)$ ), then it can overcome the double gap and fill the rows $[b] \times\{a+1\}$ and $[b] \times\{a+2\}$. Finally, if there are no further double gaps in the rows above $[b] \times[a+2]$, then the infected set can grow vertically until it fills $[b]^{2}$ (see Figure 2).

It is not hard to show that such a "detour" is less probable than the event $D$ defined above. However, Gravner and Holroyd showed that if $a$ and $b$ are 


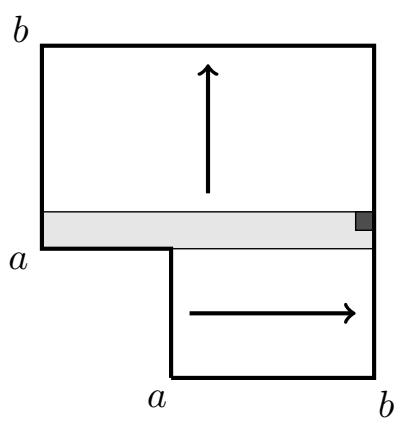

FiguRE 2. An alternative way of filling a rectangle. The light gray region is unoccupied and the dark gray square represents a single infected site. The arrows depict the growth of the infected set across regions with no double gaps.

both on the order of $1 / p$ and $b-a=O(1 / \sqrt{p})$, then these detours (or, more precisely, sequences of such detours) are both probable and numerous enough that (2.1) holds.

2.2. Higher Dimensions. Now we will describe the proof of the upper bound on $p_{c}\left([n]^{d}, r\right)$ given in [5] and discuss how we will adapt it to prove Theorem 1.4.

In order to prove the upper bound in Theorem 1.3, Balogh, Bollobás, and Morris [5] also used the notion of a critical droplet. They observed that it follows from the results in [11] that a critical droplet for $r$-neighbor percolation in $[n]^{d}$ is a $d$-dimensional box whose diameter is about $\log n$. As a heuristic justification for this, let $X_{L}$ denote the number of internally spanned cubes of diameter $L$ in $[n]^{d}$. It is shown in [11] that if $L$ is in a certain range, then the probability that a cube of diameter $L$ is internally spanned is (very roughly) $e^{-L}$. Thus, if $L \approx d \log n$, then $\mathbb{E} X_{L} \approx n^{d+o(1)} e^{-L}=\Theta(1)$, which suggests that the "critical diameter" is indeed on the order of $\log n$.

Suppose, then, that a cube $T_{0} \cong[\log n]^{d}$ is internally spanned. Under what circumstances is it likely that the infected set can grow from $T_{0}$ ? In particular, when will the infected set grow to fill the $(d-1)$-dimensional "layer" that is adjacent to $T_{0}$ in a given direction? Choose a direction and let $S_{1}$ denote the two layers adjacent to $T_{0}$ in this direction. Observe that $S_{1} \cong[\log n]^{d-1} \times[2]$. Crucially, because each site in the layer of $S_{1}$ adjacent to $T_{0},[\log n]^{d-1} \times\{1\}$, already has an infected neighbor in $T_{0}$, each such site requires only $r-1$ additional infected neighbors in $S_{1}$ in order to become infected. In contrast, sites in the other layer of $S_{1}$, $[\log n]^{d-1} \times\{2\}$, still require $r$ infected neighbors.

Therefore, it makes sense to consider percolation inside $S_{1}$, where each site in the first layer has infection threshold $r-1$ and each site in the second layer has threshold $r$. When is it likely that the first layer of $S_{1}$ will percolate? By applying the same heuristic argument as above to $S_{1}$, we see that a good 
candidate for a critical droplet in $S_{1}$ is a set of the form $[\log \log n]^{d-1} \times[2]$. (Here, the term "critical droplet" has a slightly different meaning: it refers to a set whose first layer, if fully infected, will with high probability infect the rest of the first layer of $S_{1}$.)

Suppose, then, that $S_{1}$ contains a set $T_{1} \cong[\log \log n]^{d-1} \times[2]$ whose first layer is fully infected. What is required for the infected sites in $T_{1}$ to fully infect the first layer of $S_{1}$ ? As before, percolation must occur in the first layer of each copy $S_{2}$ of $[\log \log n]^{d-2} \times[2]^{2}$ that is adjacent to $T_{1}$ (and contained in $S_{1}$ ). Note, however, that sites in the first layer of $S_{2}$, $[\log \log n]^{d-2} \times\{(1,1)\}$, need only $r-2$ infected neighbors in $S_{2}$, because each such site has one infected neighbor in $T_{0}$ and another in $T_{1}$. In contrast, sites in the other layers of $S_{2}$ still require $r$ infected neighbors in $S_{2}$ in order to become infected. ${ }^{1}$

Iterating this argument leads us to consider the probability of percolation in a set of the form $\left[\log _{(r-2)}(n)\right]^{d-r+2} \times[2]^{r-2}$, where all sites in $\left[\log _{(r-2)}(n)\right]^{d-r+2} \times\{(1, \ldots, 1)\}$ have threshold 2 and all other sites have threshold $r$. By induction, if it is likely that all sites in $\left[\log _{(r-2)}(n)\right]^{d-r+2} \times$ $\{(1, \ldots, 1)\}$ become infected, then percolation is likely to occur in $[n]^{d}$.

Balogh, Bollobás, and Morris bounded the probability that percolation occurs in $\left[\log _{(r-2)}(n)\right]^{d-r+2} \times[2]^{r-2}$ in much the same way that Holroyd bounded the probability of 2-neighbor percolation in $[n]^{2}$. Let $B \gg a$ and suppose that a cube $K \cong[a]^{d-r+2} \times \mathbf{1}^{r-2}$ is fully infected. In order to estimate the probability that $[B]^{d-r+2} \times \mathbf{1}^{r-2}$ becomes infected, we would like a fairly simple sufficient condition for all of the sites in a layer adjacent to $K$ (for example, $[a]^{d-r+1} \times\{a+1\} \times \mathbf{1}^{r-2}$ ) to become infected. Let $U_{i}$ denote the event that $[a]^{d-r+1} \times\{i\} \times \mathbf{1}^{r-2}$ is occupied and, for each $j \in[r-2]$, let $V_{i}^{(j)}$ denote the event that $[a]^{d-r+1} \times\{i\} \times\left(\mathbf{1}^{r-2}+e_{j}\right)$ is occupied. Observe that because each site in $[a]^{d-r+1} \times\{a+1\} \times \mathbf{1}^{r-2}$ has threshold 2 and already has an infected neighbor in $[a]^{d-r+2} \times \mathbf{1}^{r-2}$, all of the sites in this layer will become infected if one of the events $U_{a+1}, U_{a+2}$, $V_{a+1}^{(1)}, \ldots, V_{a+1}^{(r-2)}$ occurs. We call the situation in which none of these events occur - or, more generally, the event

$$
\neg\left(U_{i} \vee U_{i+1} \vee V_{i}^{(1)} \vee \cdots \vee V_{i}^{(r-2)}\right)
$$

for any $i \in[B]$-an $L$-gap. ${ }^{2}$ (Note that when $d=r=2$, an $L$-gap is simply a double gap.) If the sequence

$$
\mathcal{E}_{d-r+2}:=\left(U_{i}\right)_{a+1 \leq i \leq B+1} \cup\left(V_{i}^{(j)}\right)_{a+1 \leq i \leq B, j \in[r-2]}
$$

\footnotetext{
${ }^{1}$ Sites in $[\log \log n]^{d-2} \times\{(2,1)\}$ actually only need $r-1$ infected neighbors in $S_{2}$, because each one has an infected neighbor in $T_{0}$, but it turns out that we lose nothing by assuming that these sites also have infection threshold $r$.

${ }^{2}$ When $d=r=3$, the sets in question are $[a] \times\{(i, 1)\},[a] \times\{(i+1,1)\}$, and $[a] \times\{(i, 2)\}$. An $L$-gap is so called because these sets form an $L$-shape when viewed from the side.
} 
contains no $L$-gaps, then the infected set will grow in direction $d-r+2$ until it reaches one face of $[B]^{d-r+2} \times \mathbf{1}^{r-2}$. We may similarly define a sequence $\mathcal{E}_{t}$ for each direction $t \in[d-r+2]$. If none of the $\mathcal{E}_{t}$ contains an $L$-gap, then the infected set will fill $[B]^{d-r+2} \times \mathbf{1}^{r-2}$.

To bound the probability of percolation from below, it suffices to show that if $B$ is (roughly) on the order of $\log _{(r-1)}(n)$, then the probability that $[B]^{d-r+2} \times \mathbf{1}^{r-2}$ is internally spanned in the manner described above is large enough that with high probability, some cube in $\left[\log _{(r-2)}(n)\right]^{d-r+2} \times \mathbf{1}^{r-2}$ of side length $B$ is internally spanned. If so, then with high probability, the rest of $\left[\log _{(r-2)}(n)\right]^{d-r+2} \times \mathbf{1}^{r-2}$ will become infected. This proves the upper bound in Theorem 1.3 for $r=2$. The upper bound for larger $r$ follows from an inductive argument that shows that the full infection of the first layer of $\left[\log _{(r-2)}(n)\right]^{d-r+2} \times[2]^{r-2}$ indeed implies $r$-neighbor percolation in $[n]^{d}$.

As mentioned above, in order to prove Theorem 1.4, we unite the techniques of [5] and [17]. Again, we consider a fully infected cube $K \cong[a]^{d-r+2} \times$ $\mathbf{1}^{r-2}$. Let $D^{\prime}$ denote the event that the infected set grows from $K$ without encountering $L$-gaps until it fills a cube $[B]^{d-r+2} \times \mathbf{1}^{r-2}$. Just as in the two-dimensional case, we seek a large class of pairwise disjoint events $E_{1}^{\prime}$, $\ldots, E_{N^{\prime}}^{\prime}$, each of which implies that $[B]^{d-r+2} \times \mathbf{1}^{r-2}$ becomes fully infected, such that for all $i$, we have $\mathbb{P}\left(E_{i}^{\prime}\right) \geq\left(c_{1} p\right)^{1 / p^{2(d-r+1)}} \mathbb{P}\left(D^{\prime}\right)$. If, moreover, we can show that $N^{\prime}=N^{\prime}(p)=\left(c_{2} / p\right)^{1 / p^{2(d-r+1)}}$ for an appropriate constant $c_{2}$, then, similarly to (2.1), we will be able to conclude that

$$
\mathbb{P}\left(\bigvee_{i=1}^{N^{\prime}} E_{i}^{\prime}\right) \geq e^{c / p^{2(d-r+1)}} \mathbb{P}\left(D^{\prime}\right)
$$

for some constant $c>0$.

Much as in [17], we consider events $E_{i}^{\prime}$ that involve small detours in the growth of the infected set. Suppose that an $L$-gap - for example, $\neg\left(U_{a+1} \vee\right.$ $U_{a+2} \vee V_{a+1}^{(1)} \vee \cdots \vee V_{a+1}^{(r-2)}$ ) - blocks the infected set from growing from the cube $K$ in direction $d-r+2$. If no $L$-gaps occur in the other sequences $\mathcal{E}_{t}$, then the infected set may be able to grow in the other $d-r+1$ directions until it fills a set of the form $[b]^{d-r+1} \times[a] \times \mathbf{1}^{r-2}$, for some $b>a$. If there is an infected site $x$ with $x_{d-r+1} \in\{a+1, a+2\}$ (for example, $(b, \ldots, b, a+$ 2) $\times \mathbf{1}^{r-2}$ ), then the infected set can overcome the $L$-gap and fill $[b]^{d-r+1} \times$ $[a+2] \times \mathbf{1}^{r-2}$. If no further $L$-gaps occur in direction $d-r+2$, then the infected set will grow in that direction until it fills the cube $[b]^{d-r+2} \times \mathbf{1}^{r-2}$.

We show that when $a$ and $b$ are both on the order of $p^{-1 /(d-r+1)}$ and $b-$ $a=O\left(p^{-1 / 2(d-r+1)}\right)$, then the number and probability of these detours (or, rather, of sequences of such detours) are both large enough that $\mathbb{P}\left(\bigvee_{i=1}^{N^{\prime}} E_{i}^{\prime}\right)$ satisfies (2.2). This yields the claimed improvement in the upper bound on $p_{c}\left([n]^{d}, 2\right)$. 
The rest of the proof of Theorem 1.4 consists of an inductive argument that is very similar to the inductive argument of [5] mentioned above, albeit with additional technical complications.

\section{Notation And Preliminaries}

In this section, we will introduce further notation and definitions, state a useful correlation inequality, and make preliminary observations.

For the most part, our notation and terminology follow that of [5]. In order to reduce clutter, we will omit floor signs throughout the paper. All logarithms are taken with base $e$.

We say that a set $S$ is occupied if it contains at least one infected site, and empty or unoccupied otherwise. If all of the sites in $S$ are infected, we say that $S$ is full.

We will denote the vector $(1, \ldots, 1) \in \mathbb{R}^{\ell}$ by $\mathbf{1}^{\ell}$. For each $j \in[\ell]$, we let $e_{j}$ denote the $j$ th standard basis vector.

Given a set $S$, we write $A \sim \operatorname{Bin}(S, p)$ to denote that the elements of $A$ are chosen from $S$ independently with probability $p$.

Harris's Lemma [19] will play an important role in the proof. We define a partial order $\leq$ on $\{0,1\}^{n}$ by writing $x \leq y$ if, for all $i \in[n], x_{i} \leq y_{i}$. We say that an event $E \subseteq\{0,1\}^{n}$ is increasing if, for $x, y \in\{0,1\}^{n}, x \in E$ and $x \leq y$ imply that $y \in E$. Given $p \in[0,1]$, let $\mathbb{P}_{p}$ denote the product measure on $\{0,1\}^{n}$ with $\mathbb{P}_{p}(i=1)=p$ for all $i \in[n]$. (We will almost always suppress the dependence on $p$ and simply write $\mathbb{P}(\cdot)$.)

Harris's Lemma. If $E$ and $F$ are increasing events in $\{0,1\}^{n}$ and $p \in[0,1]$, then

$$
\mathbb{P}_{p}(E \cap F) \geq \mathbb{P}_{p}(E) \mathbb{P}_{p}(F) .
$$

We conclude this section by discussing properties of the functions $\beta_{k}$ and $g_{k}$ defined in (1.1) and (1.2), respectively. Given $p \in(0,1)$, we let

$$
q=-\log (1-p) .
$$

Note that for $p$ sufficiently small, we have

$$
p \leq q \leq p+p^{2} \leq 2 p .
$$

Equation (3.1) allows us to write

$$
\beta_{k}\left(1-(1-p)^{n}\right)=e^{-g_{k}(n q)} .
$$

We also observe that (1.1) implies that

$$
\beta_{k}(u)^{2}=\left(1-(1-u)^{k}\right) \beta_{k}(u)+u(1-u)^{k} .
$$

Straightforward calculations show that for all $k, \beta_{k}$ is positive, continuous, increasing, and differentiable on $(0,1)$ and $g_{k}$ is positive, continuous, decreasing, and differentiable on $(0, \infty)$.

We will need a further result about the behavior of $g_{k}$. 
Proposition 3.1. For all $k \geq 1$ and all $z \geq 1$,

$$
\left|g_{k}^{\prime}(z)\right| \leq \frac{1}{2}
$$

The proof of Proposition 3.1 is given in the Appendix.

\section{Percolation in an Auxiliary Bootstrap Structure}

In this section, we will state the key auxiliary result, Theorem 4.1, that we will use to prove Theorem 1.4. We will also define the important notion of an $L$-gap and prove a lower bound on the probability that no $L$-gaps occur in a sequence of independent events.

In Section 2.2, we related the probability of $r$-neighbor percolation in $[n]^{d}$ to the probability of percolation in a set of the form $\left[\log _{(r-2)}(n)\right]^{d-r+2} \times[2]^{r-2}$ in which not all vertices have the same infection threshold. So, in order to prove Theorem 1.4, we will consider an alternative "bootstrap structure" of this form. A bootstrap structure is an ordered pair $\left(G,(r(v))_{v \in V(G)}\right)$, where $G$ is a graph and $r: V(G) \rightarrow \mathbb{N}$. Given a vertex $v$, the value $r(v)$ is called the threshold of $v$. This means that if we consider bootstrap percolation in $\left(G,(r(v))_{v \in V(G)}\right)$ and let $A_{0}=A$ as before, then we have

$$
A_{t+1}=A_{t} \cup\left\{v:\left|N(v) \cap A_{t}\right| \geq r(v)\right\}
$$

for each $t \geq 0$.

Let $B\left([n]^{d}, r\right)$ denote the usual $r$-neighbor bootstrap structure on $[n]^{d}$. The auxiliary bootstrap structure that we will use was defined in [5]. Let $C^{*}\left([n]^{d} \times[2]^{\ell}, r\right)$ be the subgraph of $\mathbb{Z}^{d+\ell}$ induced by $[n]^{d} \times[2]^{\ell}$ in which all vertices of the form $\left(a_{1}, \ldots, a_{d}\right) \times \mathbf{1}^{\ell}$ have threshold $r$ and all other vertices have threshold $r+\ell$. Note that when $\ell=0$, this structure is the same as $B\left([n]^{d}, r\right)$.

Recall that $A$ denotes the set of initially infected vertices and that $[A]$ denotes the closure of $A$, the set of vertices that ultimately become infected. We say that $A$ semi-percolates in $C^{*}\left([n]^{d} \times[2]^{\ell}, r\right)$ if $[A] \supseteq[n]^{d} \times \mathbf{1}^{\ell}$. We say that a set $S$ is internally semi-spanned if $[S \cap A] \supseteq S \cap\left([n]^{d} \times \mathbf{1}^{\ell}\right)$.

In order to prove Theorem 1.4, we will prove a result about the probability of semi-percolation in $C^{*}\left([n]^{d} \times[2]^{\ell}, r\right)$. Before we can state it, we need additional notation.

Letting $A \sim \operatorname{Bin}\left([n]^{d} \times[2]^{\ell}, p\right)$, we set

$$
P(n, d, \ell, r, p):=\mathbb{P}\left(A \text { semi-percolates in } C^{*}\left([n]^{d} \times[2]^{\ell}, r\right)\right) .
$$

(The quantity $P(n, d, \ell, r, p)$ was originally defined in [5]. The definition given here is slightly simpler.)

Next, we define several important constants. For all $d \geq 2$ and $\ell \geq 0$, let

$$
\zeta(d, \ell)=e^{-(\ell+2) 2^{2 d-1}}\left(1-e^{-1}\right)^{2 d}
$$

and let

$$
\gamma(d, \ell)=\zeta(d, \ell) e^{-d(d-1) 2^{2 d-4}}
$$


Observe that for all $d \geq 2$ and $\ell \geq 0$,

$$
\gamma(d, \ell) \leq \gamma(2,0)=e^{-18}\left(1-e^{-1}\right)^{4}<10^{-8} .
$$

Finally, given $d \geq r \geq 2$ and $\ell \geq 0$, let

$$
c_{d, \ell, r}= \begin{cases}\gamma(d, \ell), & r=2, \\ \gamma(d-r+2, \ell+r-2)\left(1-\sum_{s=0}^{r-3} 2^{-r+s+1}\right), & r \geq 3 .\end{cases}
$$

We observe for later use that (4.5) implies that

$$
c_{d, \ell, r}=c_{d-1, \ell+1, r-1}-2^{-r+1} \gamma(d-r+2, \ell+r-2) .
$$

We are at last ready to state our auxiliary result about semi-percolation in $C^{*}\left([n]^{d} \times[2]^{\ell}, r\right)$.

Theorem 4.1. Let $d \geq r \geq 2$, let $\ell \geq 0$, and let $c_{d, \ell, r}$ be as in (4.5). If

$$
p \geq\left(\frac{\lambda(d+\ell, \ell+r)}{\log _{(r-1)}(n)}-\frac{c_{d, \ell, r}}{\left(\log _{(r-1)}(n)\right)^{3 / 2}}\right)^{d-r+1},
$$

then

$$
P(n, d, \ell, r, p) \rightarrow 1
$$

as $n \rightarrow \infty$.

In Section 6, we will show that if $p$ satisfies (4.7) for $d, \ell, r$, and $n$, then it also does so for $d-1, \ell+1, r-1$, and (roughly) $\log n$. So, by induction, if the bound on $p$ in (4.7) is sufficient for semi-percolation in $C^{*}\left(\left[\log _{(r-2)}(n)\right]^{d-r+2} \times[2]^{r-2}, 2\right)$, then it is also sufficient for percolation in $B\left([n]^{d}, r\right)$. Observe also that in order to prove Theorem 1.4, it is enough to apply Theorem 4.1 in the case when $\ell=0$ (cf. (1.4)).

Now let us define the notion of an $L$-gap. For $m \geq-1$ and $\ell \geq 0$, let $\mathcal{E}=\left(U_{i}\right)_{i \in[m+1]} \cup\left(V_{i}^{(j)}\right)_{i \in[m], j \in[\ell]}$ be a sequence of events. An $L$-gap in $\mathcal{E}$ is an event of the form

$$
\neg\left(U_{i} \vee U_{i+1} \vee V_{i}^{(1)} \vee \ldots \vee V_{i}^{(\ell)}\right)
$$

for some $i \in[m]$. (As mentioned in Section 2.2, $L$ is not a variable, but rather refers to the shape of an $L$-gap when $d=2$ and $\ell=1$.) In this paper, the events in the sequence $\mathcal{E}$ will all be of the form "a certain set of sites is occupied". Thus, an $L$-gap in $\mathcal{E}$ will mean that a certain collection of sets are all unoccupied. In particular, as was the case in Section 2.2, an $L$-gap will block the set of infected sites from growing in a specific direction.

We will need a lower bound on the probability that no $L$-gaps occur in a sequence of independent events. We can express this bound in terms of the function $\beta_{k}$ defined in (1.1). (Similar statements were proved in [5, Lemma 6] and [17, Proposition 10].) 
Lemma 4.2. Let $m \geq-1$ and $\ell \geq 0$ be integers and let $u_{1}, \ldots, u_{m+1} \in$ $(0,1)$. Let

$$
\mathcal{E}_{m+1}:=\left(U_{i}\right)_{i \in[m+1]} \cup\left(V_{i}^{(j)}\right)_{i \in[m], j \in[\ell]}
$$

be a sequence of independent events such that for each $i$, the events $U_{i}, V_{i}^{(1)}$, $\ldots, V_{i}^{(\ell)}$ each occur with probability $u_{i}$. Let $\mathbf{u}=\left(u_{i}\right)_{i=1}^{m+1}$ and let $L_{\ell}(m, \mathbf{u})$ denote the probability that no $L$-gap occurs in $\mathcal{E}_{m+1}$. If the sequence $\left(u_{i}\right)_{i=1}^{m+1}$ is increasing in $i$, then

$$
L_{\ell}(m, \mathbf{u}) \geq \prod_{i=1}^{m+1} \beta_{\ell+1}\left(u_{i}\right)
$$

In order to prove Lemma 4.2, we need another result about $\beta_{k}$.

Lemma 4.3. If $0 \leq u \leq v \leq 1$, then

$$
\left(1-(1-u)^{k}\right) \beta_{k}(v)+(1-u)^{k} v \geq \beta_{k}(u) \beta_{k}(v) .
$$

Proof. For $0 \leq u \leq v \leq 1$, define

$$
h(u, v)=\left(1-(1-u)^{k}\right) \beta_{k}(v)+(1-u)^{k} v-\beta_{k}(u) \beta_{k}(v) .
$$

and observe that we are done if we can show that $h(u, v) \geq 0$ for $u \leq v$. By (3.3),

$$
\beta_{k}(u) h(u, v)=(1-u)^{k}\left(v \beta_{k}(u)-u \beta_{k}(v)\right) .
$$

Equivalently,

$$
\frac{\beta_{k}(u)}{u v} h(u, v)=(1-u)^{k}\left(\frac{\beta_{k}(u)}{u}-\frac{\beta_{k}(v)}{v}\right),
$$

so it is enough to show that $\beta_{k}(u) / u$ is decreasing on $(0,1)$. Let $B=(1-$ $\left.(1-u)^{k}\right) / u$ and let $C=(1-u)^{k} / u$. By $(3.3), \beta_{k}(u) / u$ is the positive root of $X^{2}-B X-C=0$. Observe that both $B$ and $C$ are decreasing in $u$. It follows that

$$
\frac{\beta_{k}(u)}{u}=\frac{B+\sqrt{B^{2}+4 C}}{2}
$$

is also decreasing, as claimed.

Proof of Lemma 4.2. For $m \in\{-1,0\}$, an $L$-gap is undefined for $\mathcal{E}_{m+1}$, so, for all $u \in(0,1)$, we may take $L_{\ell}(-1, \mathbf{u})=L_{\ell}(0, \mathbf{u})=1$. Then, because $\beta_{\ell+1}:(0,1) \rightarrow(0,1)$, the result holds for $m \in\{-1,0\}$.

Let $m \geq 0$ and suppose that the result holds for values smaller than $m+$ 1. Observe that $\mathcal{E}_{m+1}$ has no $L$-gaps if (i) at least one of the events $U_{1}$, $V_{1}^{(1)}, \ldots, V_{1}^{(\ell)}$ occurs and $\left(U_{i}\right)_{2 \leq i \leq m+1} \cup\left(V_{i}^{(j)}\right)_{2 \leq i \leq m, j \in[\ell]}$ has no $L$-gaps, or (ii) none of these events occur, but the event $U_{2}$ occurs and $\left(U_{i}\right)_{3 \leq i \leq m+1} \cup$ $\left(V_{i}^{(j)}\right)_{3 \leq i \leq m, j \in[\ell]}$ has no $L$-gaps. Hence, by induction,

$$
L_{\ell}(m, \mathbf{u}) \geq\left(1-\left(1-u_{1}\right)^{\ell+1}\right) \prod_{i=2}^{m+1} \beta_{\ell+1}\left(u_{i}\right)+\left(1-u_{1}\right)^{\ell+1} u_{2} \prod_{i=3}^{m+1} \beta_{\ell+1}\left(u_{i}\right) \text {. }
$$


Because $u_{1} \leq u_{2}$, Lemma 4.3 implies that

$$
\left(1-\left(1-u_{1}\right)^{\ell+1}\right) \beta_{\ell+1}\left(u_{2}\right)+\left(1-u_{1}\right)^{\ell+1} u_{2} \geq \beta_{\ell+1}\left(u_{1}\right) \beta_{\ell+1}\left(u_{2}\right) .
$$

Combining this with the right-hand side of (4.8) yields the claimed inequality.

\section{Proof of Theorem 4.1 For $r=2$}

Our aim in this section is to prove a result that implies Theorem $4.1 \mathrm{in}$ the case $r=2$.

Lemma 5.1. Let $d \geq 2$, let $\ell \geq 0$, and let $\gamma(d, \ell)$ be as in (4.3). If $c$ is a constant such that

$$
0<c<\frac{3}{2} \gamma(d, \ell)
$$

and

$$
p \geq\left(\frac{\lambda(d+\ell, \ell+2)}{\log n}-\frac{c}{(\log n)^{3 / 2}}\right)^{d-1},
$$

then

$$
P(n, d, \ell, 2, p) \rightarrow 1
$$

as $n \rightarrow \infty$.

Remark 5.2. By (4.5), $c_{d, \ell, 2}$ certainly satisfies (5.1) for all $d \geq 2$ and $\ell \geq 0$.

Here is a sketch of the proof of Lemma 5.1. Below, we will define an event $\mathcal{D}_{a}^{b}$ that implies that if $[a]^{d} \times \mathbf{1}^{\ell}$ is internally spanned, then the infected set grows to fill $[b-1]^{d} \times \mathbf{1}^{\ell}$ "diagonally", i.e., by iteratively filling sets of the form $[i]^{d} \times \mathbf{1}^{\ell}$. The main step in the proof of the upper bound on $p_{c}\left([n]^{d}, 2\right)$ given in [5] amounts to a lower bound on $\mathbb{P}\left(\mathcal{D}_{a}^{b}\right)$. In order to prove a stronger bound on $p_{c}\left([n]^{d}, 2\right)$, we will define an event $\mathcal{T}_{a}^{\mathbf{b}}$ (the vector superscript is explained below) that implies that the infected set grows from $[a]^{d} \times \mathbf{1}^{\ell}$ to $[b]^{d} \times \mathbf{1}^{\ell}$ not diagonally but via a "detour".

We will show that $\mathcal{T}_{a}^{\mathbf{b}}$ is not too much less probable than $\mathcal{D}_{a}^{b}$ (Lemma 5.6). As the infected set grows, it may make a detour and then resume diagonal growth several times. So, we think of the growth of the infected set as diagonal growth interrupted by a sequence of detours. We will show that different "growth sequences" of this sort are disjoint events (Lemma 5.7) and that the number of growth sequences is fairly large (Lemma 5.8). Furthermore, we will use these results to show that if $p$ satisfies (5.2), then with high probability some cube of the form $[B]^{d} \times[2]^{\ell}$, where $B=B(p)$ is sufficiently large, is internally semi-spanned. Finally, we will show that with high probability, such a fully infected cube leads to semi-percolation in $C^{*}\left([n]^{d} \times[2]^{\ell}, 2\right)$, which will complete the proof of Lemma 5.1.

Recall the definition of an $L$-gap from Section 4 and recall that for $j \in[\ell]$, $e_{j}$ denotes the $j$ th standard basis vector of $\mathbb{R}^{\ell}$. For all $i, s \in \mathbb{N}$ and $t \in[d]$, let

$$
U_{i}(t, s)=\left\{[s]^{t-1} \times\{i\} \times[s]^{d-t} \times \mathbf{1}^{\ell} \text { is occupied }\right\},
$$


and for all $j \in[\ell]$, let

$$
V_{i}^{(j)}(t, s)=\left\{[s]^{t-1} \times\{i\} \times[s]^{d-t} \times\left(\mathbf{1}^{\ell}+e_{j}\right) \text { is occupied }\right\} .
$$

Let $\mathcal{D}_{a}^{b}$ be the event that for each $t \in[d]$, the sequence

$$
\left(U_{i}(t, i-1)\right)_{a+1 \leq i \leq b} \cup\left(V_{i}^{(j)}(t, i-1)\right)_{a+1 \leq i \leq b-1, j \in[\ell]}
$$

has no $L$-gaps.

The next result shows that, as mentioned above, the event $\mathcal{D}_{a}^{b}$ means that the infected set grows "diagonally" from $[a]^{d} \times \mathbf{1}^{\ell}$ to $[b-1]^{d} \times \mathbf{1}^{\ell}$. Recall that we say that a set $S$ is internally semi-spanned if $[S \cap A] \supseteq S \cap\left([n]^{d} \times \mathbf{1}^{\ell}\right)$.

Lemma 5.3. If $[a]^{d} \times[2]^{\ell}$ is internally semi-spanned and $\mathcal{D}_{a}^{b}$ occurs, then $[b-1]^{d} \times[2]^{\ell}$ is internally semi-spanned.

Proof. We will show that if $[a]^{d} \times \mathbf{1}^{\ell}$ is internally spanned and $\mathcal{D}_{a}^{b}$ occurs, then for each $i$ with $a+1 \leq i \leq b-1$, the set $[i]^{d} \times \mathbf{1}^{\ell}$ is internally spanned. We assume inductively that $[i-1]^{d} \times \mathbf{1}^{\ell}$ is internally spanned. By hypothesis, for each $t \in[d]$, the sequence in (5.5) does not have an $L$-gap at $i$, which means that for each $t$, all of the sites in $[i-1]^{t-1} \times\{i\} \times[i-1]^{d-t} \times \mathbf{1}^{\ell}$ become infected. (Note that each such site already has one infected neighbor in $[i-1]^{d} \times \mathbf{1}^{\ell}$.) Therefore, all of $[i]^{d} \times \mathbf{1}^{\ell}$ becomes infected. This completes the proof.

Let

$$
G_{a}^{b}=\exp \left[-\sum_{i=a}^{b-1} g_{\ell+1}\left(i^{d-1} q\right)\right],
$$

where $q$ is as defined in (3.1). Observe that if $a<b<c$, then

$$
G_{a}^{c}=G_{a}^{b} G_{b}^{c}
$$

Lemma 5.4. For all $d \geq 2$ and all $b>a \geq 2$,

$$
\mathbb{P}\left(\mathcal{D}_{a}^{b}\right) \geq\left(G_{a}^{b}\right)^{d} \text {. }
$$

Proof. Observe from (5.3) and (5.4) that, for each $i$ and $t$, the events $U_{i}(t, i-$ $1), U_{i+1}(t, i), V_{i}^{(1)}(t, i-1), \ldots, V_{i}^{(\ell)}(t, i-1)$ concern pairwise disjoint sets of sites and are therefore independent. Furthermore, the probability that these events occur is increasing in $i$. Hence, the sequence (5.5) satisfies the hypotheses of Lemma 4.2. This and (3.2) imply that

$$
\begin{aligned}
\mathbb{P}\left(\mathcal{D}_{a}^{b}\right) & \geq\left(\prod_{i=a+1}^{b} \beta_{\ell+1}\left(1-(1-p)^{(i-1)^{d-1}}\right)\right)^{d} \\
& =\exp \left[-d \sum_{i=a+1}^{b} g_{\ell+1}\left((i-1)^{d-1} q\right)\right] \\
& =\left(G_{a}^{b}\right)^{d}
\end{aligned}
$$

as claimed. 
Now we will define the "detour" mentioned above. In [17], Gravner and Holroyd defined an event $\mathcal{T}_{a}^{b}$ that describes another way for the infected set to grow from $[a]^{2}$ to $[b]^{2}$. (A simplified version of $\mathcal{T}_{a}^{b}$ is shown in Figure 2.) If the rows $[b-1] \times\{a+2\}$ and $[b-1] \times\{a+3\}$ are empty, then the infected set is prevented from growing vertically. However, if there are no double gaps in the columns to the right of $[a]^{2}$, then the infected set grows horizontally until it fills the rectangle $[b] \times[a+1]$. If the site $(b, a+3)$ is infected, then the infected set overcomes the double gap and resumes vertical growth, ultimately filling $[b]^{2}$.

We will define a similar event $\mathcal{T}_{a}^{\mathbf{b}}$, where $\mathbf{b}:=\left\{b_{1}, \ldots, b_{d-1}\right\}$. In this event, an $L$-gap prevents the fully infected cube $[a+1]^{d} \times \mathbf{1}^{\ell}$ from growing in direction $d$ (parts (ii), (iii), and (v) of the definition below). However, the infected set continues to grow in the other $d-1$ directions (parts (vi) and (vii)) until it meets the infected site $\left\{b_{1}, \ldots, b_{d-1}, a+3\right\} \times \mathbf{1}^{\ell}$ (part (iv)). This site allows the infected set to overcome the $L$-gap. Finally, letting $b=\max \left\{b_{i}: i \in[d-1]\right\}$, the infected set continues to grow in direction $d$ until it fills a cube of side length $b$ (parts (viii) and (ix)).

Recall the definitions of the events $U_{i}(t, s)$ and $V_{i}^{(j)}(t, s)$ from (5.3) and (5.4), respectively. Let $a, b_{1}, \ldots, b_{d-1}$ be such that $b:=\max \left\{b_{i}: i \in[d-1]\right\}$ satisfies $b \geq a+4$ and let $\mathbf{b}=\left\{b_{1}, \ldots, b_{d-1}\right\}$. Define $\mathcal{T}_{a}^{\mathbf{b}}$ to be the event that all of the following hold (see Figure 3, which depicts the case $d=2, \ell=1$ ).

(i) For all $t \in[d]$, the cuboid $[a-1]^{t-1} \times\{a+1\} \times[a-1]^{d-t} \times \mathbf{1}^{\ell}$ is occupied.

(ii) The cuboid $[b]^{d-1} \times\{a+2\} \times \mathbf{1}^{\ell}$ is empty.

(iii) For all $j \in[\ell]$, the cuboid $[b]^{d-1} \times\{a+2\} \times\left(\mathbf{1}^{\ell}+e_{j}\right)$ is empty.

(iv) The site $\left\{b_{1}, \ldots, b_{d-1}, a+3\right\} \times \mathbf{1}^{\ell}$ is infected.

(v) The cuboid $[b]^{d-1} \times\{a+3\} \times \mathbf{1}^{\ell}$ contains no other infected sites.

(vi) For all $t \neq d$, the sequence

$$
\left(U_{i}(t, a+1)\right)_{a+2 \leq i \leq b-1} \cup\left(V_{i}^{(j)}(t, a+1)\right)_{a+2 \leq i \leq b-2, j \in[\ell]}
$$

has no $L$-gaps.

(vii) For all $t \neq d$, the cuboid $[a+1]^{t-1} \times\{b\} \times[a+1]^{d-t} \times \mathbf{1}^{\ell}$ is occupied.

(viii) The sequence

$$
\left(U_{i}(d, b)\right)_{a+4 \leq i \leq b-1} \cup\left(V_{i}^{(j)}(d, b)\right)_{a+4 \leq i \leq b-2, j \in[\ell]}
$$

has no $L$-gaps.

(ix) The cuboid $[b]^{d-1} \times\{b\} \times \mathbf{1}^{\ell}$ is occupied.

Lemma 5.5. (i) Events (i)-(ix) in the definition of $\mathcal{T}_{a}^{\mathbf{b}}$ are independent.

(ii) If $[a-1]^{d} \times[2]^{\ell}$ is internally semi-spanned and $\mathcal{T}_{a}^{\mathbf{b}}$ occurs, then $[b]^{d} \times[2]^{\ell}$ is also internally semi-spanned.

Proof. (i) This follows from the fact that events (i)-(ix) in the definition of $\mathcal{T}_{a}^{\mathrm{b}}$ concern pairwise disjoint sets of sites. Indeed, all of the sites in the sets described in parts (i), (vi), and (vii) have $d$ th coordinate at most $a+1$. 


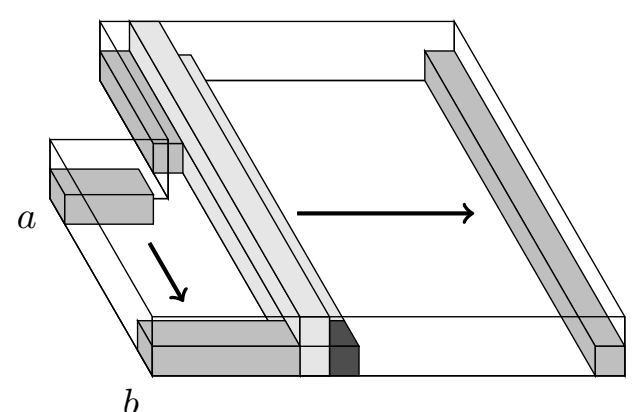

Figure 3. The event $\mathcal{T}_{a}^{\mathbf{b}}$ for $d=2$ and $\ell=1$. The gray regions are occupied (parts (i), (vii), and (ix) of the definition) and the light gray regions are unoccupied (parts (ii), (iii), and $(\mathrm{v})$ ). The dark gray cube is an infected site (part (iv)). The arrows depict the growth of the infected set across regions with no $L$-gaps (parts (vi) and (viii)).

Moreover, all of the sites in the sets described by the events $U_{i}(t, a+1)$ and $V_{i}^{(j)}(t, a+1)$ have $i$ th coordinate $t$. Similarly, all of the sites in the sets described in parts (ii), (iii), (iv), and (v) have $d$ th coordinate in $\{a+2, a+3\}$, and it is easy to see that these four sets are pairwise disjoint. Finally, all of the sites in parts (viii) and (ix) have $d$ th coordinate at least $a+4$, and it is again easy to see that the sets mentioned in these parts are pairwise disjoint.

(ii) If $[a-1]^{d} \times[2]^{\ell}$ is internally semi-spanned, then part (i) implies that each set of the form $[a-1]^{t-1} \times\{a\} \times[a-1]^{d-t} \times \mathbf{1}^{\ell}$ becomes fully infected. This in turn guarantees that all of $[a]^{d} \times \mathbf{1}^{\ell}$, and then all of $[a+1]^{d} \times \mathbf{1}^{\ell}$, becomes infected. Parts (vi) and (vii) then guarantee that $[b]^{d-1} \times[a+1] \times[2]^{\ell}$ is internally semi-spanned. Finally, parts (iv), (viii), and (ix) imply that $[b]^{d} \times[2]^{\ell}$ is internally semi-spanned.

Now we will show that if $b=\max \left\{b_{i}: i \in[d-1]\right\}$, then $\mathcal{T}_{a}^{\mathbf{b}}$ is not too much less probable than $\mathcal{D}_{a}^{b}$. It will be convenient to compare $\mathbb{P}\left(\mathcal{T}_{a}^{\mathbf{b}}\right)$ not to $\mathbb{P}\left(\mathcal{D}_{a}^{b}\right)$ but to $G_{a}^{b}$.

Lemma 5.6. Let $d \geq 2$, let $\ell \geq 0$, and let $\zeta=\zeta(d, \ell)$ be the constant defined in (4.2). If $p>0$ is sufficiently small, if $a, b_{1}, \ldots, b_{d-1}$ are integers in the interval $\left[p^{-1 /(d-1)}+1,4 p^{-1 /(d-1)}\right]$ such that $b:=\max \left\{b_{i}: i \in[d-1]\right\}$ satisfies $b \geq a+4$, and if $\mathbf{b}=\left\{b_{1}, \ldots, b_{d-1}\right\}$, then

$$
\mathbb{P}\left(\mathcal{T}_{a}^{\mathbf{b}}\right) \geq \zeta p \exp \left[-p d(b-a)\left(b^{d-1}-a^{d-1}\right)\right]\left(G_{a}^{b}\right)^{d} .
$$

The key to the proof of Lemma 5.6 is that if $p$ is sufficiently small and $s$ is on the order of $p^{-1 /(d-1)}$, then $(1-p)^{s^{d-1}}$ is bounded away from both 0 and 1 . 
Proof of Lemma 5.6. By Lemmas 4.2 and 5.5(i) and the definition of $\mathcal{T}_{a}^{\mathbf{b}}$,

$$
\begin{aligned}
\mathbb{P}\left(\mathcal{T}_{a}^{\mathbf{b}}\right) \geq( & \left.-(1-p)^{(a-1)^{d-1}}\right)^{d}(1-p)^{b^{d-1}}(1-p)^{\ell b^{d-1}} p(1-p)^{b^{d-1}-1} \\
& \times \beta_{\ell+1}\left(1-(1-p)^{(a+1)^{d-1}}\right)^{(d-1)(b-a-2)}\left(1-(1-p)^{(a+1)^{d-1}}\right)^{d-1} \\
& \times \beta_{\ell+1}\left(1-(1-p)^{b^{d-1}}\right)^{b-a-4}\left(1-(1-p)^{b^{d-1}}\right) .
\end{aligned}
$$

Because $b>a$, we have

$$
\begin{aligned}
\mathbb{P}\left(\mathcal{T}_{a}^{\mathbf{b}}\right) \geq p & (1-p)^{(\ell+2) b^{d-1}-1}\left(1-(1-p)^{(a-1)^{d-1}}\right)^{2 d} \\
& \times \beta_{\ell+1}\left(1-(1-p)^{(a+1)^{d-1}}\right)^{(d-1)(b-a)} \beta_{\ell+1}\left(1-(1-p)^{b^{d-1}}\right)^{b-a}
\end{aligned}
$$

If $x$ is sufficiently small, then $e^{-x} \geq 1-x \geq e^{-2 x}$. So, because $b \leq 4 p^{-1 /(d-1)}$ and $a-1 \geq p^{-1 /(d-1)}$, if $p$ is sufficiently small, then

$$
(1-p)^{(\ell+2) b^{d-1}-1}\left(1-(1-p)^{(a-1)^{d-1}}\right)^{2 d} \geq e^{-(\ell+2) 2^{2 d-1}}\left(1-e^{-1}\right)^{2 d}=\zeta .
$$

When we plug this into (5.8) and use (3.2), we see that

$$
\begin{aligned}
\mathbb{P}\left(\mathcal{T}_{a}^{\mathbf{b}}\right) & \geq \zeta p \beta_{\ell+1}\left(1-(1-p)^{(a+1)^{d-1}}\right)^{(d-1)(b-a)} \beta_{\ell+1}\left(1-(1-p)^{b^{d-1}}\right)^{b-a} \\
& =\zeta p \exp \left[-(d-1)(b-a) g_{\ell+1}\left((a+1)^{d-1} q\right)-(b-a) g_{\ell+1}\left(b^{d-1} q\right)\right] .
\end{aligned}
$$

Finally, since $g_{\ell+1}$ is decreasing, we have

$$
\mathbb{P}\left(\mathcal{T}_{a}^{\mathbf{b}}\right) \geq \zeta p \exp \left[-d(b-a) g_{\ell+1}\left(a^{d-1} q\right)\right] .
$$

Observe from (5.6) that

$$
G_{a}^{b}=\exp \left[-\sum_{i=a}^{b-1} g_{\ell+1}\left(i^{d-1} q\right)\right] \leq \exp \left[-(b-a) g_{\ell+1}\left(b^{d-1} q\right)\right] .
$$

Thus, we may rewrite (5.9) as

$$
\mathbb{P}\left(\mathcal{T}_{a}^{\mathbf{b}}\right) \geq \zeta p \exp \left[-d(b-a)\left(g_{\ell+1}\left(a^{d-1} q\right)-g_{\ell+1}\left(b^{d-1} q\right)\right)\right]\left(G_{a}^{b}\right)^{d} .
$$

Now

$$
g_{\ell+1}\left(a^{d-1} q\right)-g_{\ell+1}\left(b^{d-1} q\right) \leq\left(b^{d-1} q-a^{d-1} q\right) \max _{x \in\left[a^{d-1} q, b^{d-1} q\right]}\left|g_{\ell+1}^{\prime}(x)\right| .
$$

Recall that $p \leq q$, which, by our assumptions on $a$ and $b$, means that $1 \leq$ $a^{d-1} q<b^{d-1} q$. So, Proposition 3.1 and the fact that $q \leq 2 p$ for $p$ sufficiently small imply that

$$
g_{\ell+1}\left(a^{d-1} q\right)-g_{\ell+1}\left(b^{d-1} q\right) \leq\left(b^{d-1}-a^{d-1}\right) q \cdot \frac{1}{2}=p\left(b^{d-1}-a^{d-1}\right) .
$$

Combining this with (5.10) gives the desired result. 
If semi-percolation occurs in $C^{*}\left([n]^{d} \times[2]^{\ell}, 2\right)$, then, as the infected set grows, it may encounter and overcome several $L$-gaps. We order the $L$-gaps by the associated value of $a$ and define $\mathbf{b}_{i}$ to be the vector associated with the $i$ th $L$-gap.

Now we will define the event that the infected set grows from $[2]^{d} \times \mathbf{1}^{\ell}$ to $[B]^{d} \times \mathbf{1}^{\ell}$ (where $B=B(p)$ is a large value to be chosen later) with periods of diagonal growth interrupted by a specified sequence of events of the form $\mathcal{T}_{a}^{\mathbf{b}}$.

For each $t \in[d]$, let $x^{(t)}=\{1\}^{t-1} \times\{2\} \times\{1\}^{d-t} \times \mathbf{1}^{\ell}$ and let $y^{(t)}=\{B\}^{t-1} \times$ $\{1\} \times\{B\}^{d-t} \times \mathbf{1}^{\ell}$. Let $m \in \mathbb{N}$ and let $2 \leq a_{1}<b_{1} \leq \ldots \leq a_{m}<b_{m}$ be such that for all $i \in[m]$, we have $b_{i}-a_{i} \geq 4$, and such that $B>b_{m}$. For each $i \in[m]$, let $\mathbf{b}_{i}=\left\{b_{i, 1}, \ldots, b_{i, d-1}\right\}$ be such that $b_{i}=\max \left\{b_{i, t}: t \in[d-1]\right\}$. Define

$$
\begin{aligned}
\mathcal{G}\left(a_{1}, \mathbf{b}_{1}, \ldots, a_{m}, \mathbf{b}_{m}\right)=\left(\{1\}^{d}\right. & \left.\times \mathbf{1}^{\ell} \text { is infected }\right) \cap\left(\bigcap_{t=1}^{d}\left(x^{(t)} \text { is infected }\right)\right) \\
& \cap \mathcal{D}_{2}^{a_{1}} \cap \mathcal{T}_{a_{1}}^{\mathbf{b}_{1}} \cap \cdots \cap \mathcal{D}_{b_{m-1}}^{a_{m}} \cap \mathcal{T}_{a_{m}}^{\mathbf{b}_{m}} \cap \mathcal{D}_{b_{m}}^{B-1} \\
& \cap\left(\bigcap_{t=1}^{d}\left(y^{(t)} \text { is infected }\right)\right) .
\end{aligned}
$$

Lemma 5.7. (i) The events in the definition of $\mathcal{G}\left(\left(a_{i}, \mathbf{b}_{i}\right)_{i=1}^{m}\right)$ are independent.

(ii) If $\mathcal{G}\left(\left(a_{i}, \mathbf{b}_{i}\right)_{i=1}^{m}\right)$ occurs then $C^{*}\left([B]^{d} \times[2]^{\ell}, 2\right)$ is internally semispanned.

(iii) Events of the form $\mathcal{G}\left(\left(a_{i}, \mathbf{b}_{i}\right)_{i=1}^{m}\right)$ are pairwise disjoint, that is, they correspond to pairwise disjoint subsets of $\{0,1\}^{B^{d} 2^{\ell}}$.

Proof. (i) It follows from the definition of $\mathcal{D}_{a}^{b}$ and from Lemma 5.5(i) that the events in the definition of $\mathcal{G}\left(\left(a_{i}, \mathbf{b}_{i}\right)_{i=1}^{m}\right)$ involve pairwise disjoint sets of sites. Thus, they are independent.

(ii) First, if all of the sites $\{1\}^{d} \times \mathbf{1}^{\ell}, x^{(1)}, \ldots, x^{(d)}$ are infected, then $[2]^{d+\ell}$ is internally semi-spanned. Next, observe that by Lemmas 5.3 and 5.5 (ii), if the events $\mathcal{D}_{2}^{a_{1}}, \mathcal{T}_{a_{1}}^{\mathbf{b}_{1}}, \ldots, \mathcal{D}_{b_{m-1}}^{a_{m}}, \mathcal{T}_{a_{m}}^{\mathbf{b}_{m}}$, and $\mathcal{D}_{b_{m}}^{B-1}$ all occur, then $[B-2]^{d} \times$ $[2]^{\ell}$ is internally semi-spanned. Finally, if all of the sites $y^{(1)}, \ldots, y^{(d)}$ are infected, then $[B]^{d} \times[2]^{\ell}$ is internally semi-spanned.

(iii) Consider two sequences $\left(a_{i}, \mathbf{b}_{i}\right)_{i=1}^{m}$ and $\left(a_{i}^{\prime}, \mathbf{b}_{i}^{\prime}\right)_{i=1}^{m}$ and the associated events $\mathcal{G}\left(\left(a_{i}, \mathbf{b}_{i}\right)_{i=1}^{m}\right)$ and $\mathcal{G}\left(\left(a_{i}^{\prime}, \mathbf{b}_{i}^{\prime}\right)_{i=1}^{m}\right)$. Recall the definitions of the events $U_{i}(t, s)$ and $V_{i}^{(j)}(t, s)$ from (5.3) and (5.4), respectively. Given $i \geq 1$, it follows from the definition of $\mathcal{D}_{a}^{b}$ and parts (ii), (iii), and (v) of the definition of $\mathcal{T}_{a}^{\mathrm{b}}$ that $a_{i}+2$ is the least $s \geq b_{i-1}$ such that the events $U_{s}(d, s-1)$, $U_{s+1}(d, s), V_{s}^{(1)}(d, s-1), \ldots, V_{s}^{(\ell)}(d, s-1)$ all do not occur. (Here, we set $b_{0}=2$.) This means that if $a_{i} \neq a_{i}^{\prime}$, then $\mathcal{G}\left(\left(a_{i}, \mathbf{b}_{i}\right)_{i=1}^{m}\right)$ and $\mathcal{G}\left(\left(a_{i}^{\prime}, \mathbf{b}_{i}^{\prime}\right)_{i=1}^{m}\right)$ are disjoint. Similarly, parts (iv) and (v) of the definition of $\mathcal{T}_{a}^{\mathbf{b}}$ imply that 
if $\mathbf{b}_{i} \neq \mathbf{b}_{i}^{\prime}$, then $\mathcal{G}\left(\left(a_{i}, \mathbf{b}_{i}\right)_{i=1}^{m}\right)$ and $\mathcal{G}\left(\left(a_{i}^{\prime}, \mathbf{b}_{i}^{\prime}\right)_{i=1}^{m}\right)$ are disjoint. Thus, the two events are disjoint unless they are identical, as claimed.

Parts (ii) and (iii) of Lemma 5.7 indicate that if we can bound from below the probability that an event of the form $\mathcal{G}\left(\left(a_{i}, \mathbf{b}_{i}\right)_{i=1}^{m}\right)$ occurs, then a union bound will give us a lower bound on the probability of semi-percolation. To this end, we wish to enumerate those sequences $\left(a_{i}, \mathbf{b}_{i}\right)_{i=1}^{m}$ that satisfy certain conditions. We will be interested in sequences such that

$$
p^{-1 /(d-1)}+1 \leq a_{1}<b_{1} \leq \ldots \leq a_{m}<b_{m} \leq 4 p^{-1 /(d-1)}
$$

and

$$
4 \leq b_{i}-a_{i} \leq p^{-1 /(2 d-2)} \quad \text { for all } i \in[m] .
$$

Let us explain these conditions. First, the lower bound on the probability of $\mathcal{T}_{a}^{\mathbf{b}}$ in Lemma 5.6 requires that $a$ and $b$ both be on the order of $p^{-1 /(d-1)}$, which corresponds to (5.11).

Second, we wish to show that there are many sequences $\left(a_{i}, \mathbf{b}_{i}\right)_{i=1}^{m}$ such that for all $i$, we have $b_{i}-a_{i} \leq K=K(p)$. What, then, should $K$ and $m$ be? Observe that (5.11) implies that $K m \leq p^{-1 /(d-1)}$. Moreover, we will show that, given $K$ and $m$, the number of sequences of the desired form is roughly $(K / m p)^{m}$, which is maximized when $K$ and $m$ have the same order of magnitude. Thus, we will take both $K$ and $m$ to be on the order of $p^{-1 /(2 d-2)}$; the former requirement is the second inequality in (5.12). Finally, $\mathcal{T}_{a}^{\mathrm{b}}$ is defined only if $b \geq a+4$.

Lemma 5.8. Let $d \geq 2$, let $\ell \geq 0$, and let $p>0$ be sufficiently small. If $\gamma=\gamma(d, \ell)$ is as in (4.3) and

$$
m=\gamma p^{-1 /(2 d-2)}
$$

then the number of sequences $\left(a_{i}, \mathbf{b}_{i}\right)_{i=1}^{m}$ satisfying (5.11) and (5.12) is at least

$$
\left(\frac{8}{\gamma p}\right)^{m}
$$

Proof. We construct sequences $\left(a_{i}, \mathbf{b}_{i}\right)_{i=1}^{m}$ satisfying (5.11) and (5.12) as follows: we start by choosing $a_{1}, \ldots, a_{m}$ such that $a_{1} \geq p^{-1 /(d-1)}+1$, such that $a_{i+1} \geq a_{i}+p^{-1 /(2 d-2)}$ for all $i \in[m-1]$, and such that $a_{m} \leq$ $4 p^{-1 /(d-1)}-p^{-1 /(2 d-2)}$. Then, for each $i$, we choose $\mathbf{b}_{i}$ as follows. First, we choose an element of $\left\{a_{i}+4, \ldots, a_{i}+p^{-1 /(2 d-2)}\right\}$ and call it $b_{i}$. Then, to complete the vector $\mathbf{b}_{i}$, we choose $d-2$ elements of $\left[b_{i}\right]$ with replacement. Observe that a sequence chosen in this way indeed satisfies (5.11) and (5.12). 
Let $\mathcal{S}$ denote the set of sequences chosen above and observe that by Stirling's approximation, if $p$ is sufficiently small, then

$$
\begin{aligned}
|\mathcal{S}| & \geq\left(\begin{array}{c}
3 p^{-\frac{1}{d-1}}-1-m p^{-\frac{1}{2 d-2}} \\
m
\end{array}\right)\left(p^{-\frac{1}{2 d-2}}-3\right)^{m} \prod_{i=1}^{m} b_{i}^{d-2} \\
& \geq\left(\frac{e(3-2 \gamma) p^{-\frac{1}{d-1}}}{m}\right)^{m}\left((1-\gamma) p^{-\frac{1}{2 d-2}}\right)^{m} \prod_{i=1}^{m} b_{i}^{d-2} .
\end{aligned}
$$

For each $i \in[m]$, we have $b_{i} \geq p^{-1 /(d-1)}$. It follows from (4.4) that

$$
|\mathcal{S}| \geq\left(\frac{e(3-5 \gamma) p^{-\frac{1}{2 d-2}}}{m}\right)^{m}\left(p^{-\frac{1}{d-1}}\right)^{m}\left(p^{-\frac{d-2}{d-1}}\right)^{m} \geq\left(\frac{8}{\gamma p}\right)^{m}
$$

This completes the proof.

Remark 5.9. Let us make two further remarks regarding Lemma 5.8. First, one might also count sequences of fewer than $m L$-gaps, but it turns out that this would not significantly affect the total. (Essentially, this is because if $M \gg m$, then $\sum_{j=1}^{m}(M / j)^{j} \leq m(M / m)^{m}$; for our purposes, the extra factor of $m$ represents a negligible increase.)

Second, recall that in part (iv) of the definition of $\mathcal{T}_{a}^{\mathbf{b}}$ we required that the site $\left(b_{1}, \ldots, b_{d-1}, a+3\right) \times \mathbf{1}^{\ell}$ be infected. One might be tempted to define $\mathcal{T}_{a}^{\mathbf{b}}$ so that the site $(b, \ldots, b, a+3) \times \mathbf{1}^{\ell}$ is infected. However, with this alternative definition, no result similar to Lemma 5.8 holds. That is, there does not exist a constant $c>0$ such that the number of sequences $\left(a_{i}, \mathbf{b}_{i}\right)_{i=1}^{m}$ satisfying (5.11) and (5.12) is at least $(c / p)^{m}$-and, as the proof of Lemma 5.10 will show, this bound is exactly what we need.

Recall the definition of $P(n, d, \ell, r, p)$ from (4.1). Now we will combine the results above to prove a lower bound on $P(B, d, \ell, 2, p)$ for $B>4 p^{-1 /(d-1)}$. Once we have done so, we will be ready to prove Lemma 5.1 .

Lemma 5.10. Let $d \geq 2$, let $\ell \geq 0$, and let $\gamma=\gamma(d, \ell)$ be as in (4.3). If $p>0$ is sufficiently small and $B>4 p^{-1 /(d-1)}$, then

$$
P(B, d, \ell, 2, p) \geq \exp \left[\frac{2 \gamma}{p^{1 /(2 d-2)}}-\frac{d \lambda(d+\ell, \ell+2)}{p^{1 /(d-1)}}\right] .
$$

Proof. Let $m$ be as in the statement of Lemma 5.8 and suppose that the sequence $\left(a_{i}, \mathbf{b}_{i}\right)_{i=1}^{m}$ satisfies (5.11) and (5.12). (By (5.11), $B>b_{m}$.) We begin by bounding $\mathbb{P}\left(\mathcal{G}\left(\left(a_{i}, \mathbf{b}_{i}\right)_{i=1}^{m}\right)\right)$ from below. By Lemma 5.7(i), we have

$$
\mathbb{P}\left(\mathcal{G}\left(\left(a_{i}, \mathbf{b}_{i}\right)_{i=1}^{m}\right)\right)=p^{2 d+1} \mathbb{P}\left(\mathcal{D}_{2}^{a_{1}}\right) \mathbb{P}\left(\mathcal{T}_{a_{1}}^{\mathbf{b}_{1}}\right) \cdots \mathbb{P}\left(\mathcal{D}_{b_{m-1}}^{a_{m}}\right) \mathbb{P}\left(\mathcal{T}_{a_{m}}^{\mathbf{b}_{m}}\right) \mathbb{P}\left(\mathcal{D}_{b_{m}}^{B-1}\right)
$$

Recall that in Lemmas 5.4 and 5.6, we bounded $\mathbb{P}\left(\mathcal{D}_{a}^{b}\right)$ and $\mathbb{P}\left(\mathcal{T}_{a}^{\mathbf{b}}\right)$, respectively, in terms of $G_{a}^{b}$. It follows from these results and (5.7) that

$$
\mathbb{P}\left(\mathcal{G}\left(\left(a_{i}, \mathbf{b}_{i}\right)_{i=1}^{m}\right)\right) \geq p^{2 d+1}\left(G_{2}^{B-1}\right)^{d} \prod_{i=1}^{m}\left(\zeta p e^{-p d\left(b_{i}-a_{i}\right)\left(b_{i}^{d-1}-a_{i}^{d-1}\right)}\right) .
$$


By the Mean Value Theorem, for each $i$, there exists $\alpha_{i} \in\left[a_{i}, b_{i}\right]$ such that

$$
b_{i}^{d-1}-a_{i}^{d-1}=\left(b_{i}-a_{i}\right)(d-1) \alpha_{i}^{d-2} .
$$

It then follows from (5.11) and (5.12) that

$$
\left(b_{i}-a_{i}\right)\left(b_{i}^{d-1}-a_{i}^{d-1}\right) \leq(d-1)\left(b_{i}-a_{i}\right)^{2} b_{i}^{d-2} \leq(d-1) 2^{d-2} p^{-1} .
$$

Plugging this into (5.14) and recalling the definition of $\gamma$ from (4.3) shows that

$$
\begin{aligned}
\mathbb{P}\left(\mathcal{G}\left(\left(a_{i}, \mathbf{b}_{i}\right)_{i=1}^{m}\right)\right) & \geq p^{2 d+1}\left(G_{2}^{B-1}\right)^{d} \prod_{i=1}^{m}\left(\zeta p e^{-d(d-1) 2^{d-2}}\right) \\
& =p^{2 d+1}\left(G_{2}^{B-1}\right)^{d}(\gamma p)^{m} .
\end{aligned}
$$

Now let $\lambda=\lambda(d+\ell, \ell+2)$ be as in (1.3). Observe that (5.6), the fact that $g_{\ell+1}$ is decreasing, and the fact that $p \leq q$ imply that

$$
\begin{aligned}
G_{2}^{B-1} & =\exp \left[-\sum_{i=2}^{B-2} g_{\ell+1}\left(i^{d-1} q\right)\right] \\
& \geq \exp \left[-\frac{1}{p^{1 /(d-1)}} \int_{0}^{\infty} g_{\ell+1}\left(z^{d-1}\right) d z\right] \\
& =\exp \left[-\frac{\lambda}{p^{1 /(d-1)}}\right]
\end{aligned}
$$

Plugging this into (5.15), we see that

$$
\mathbb{P}\left(\mathcal{G}\left(\left(a_{i}, \mathbf{b}_{i}\right)_{i=1}^{m}\right)\right) \geq p^{2 d+1}(\gamma p)^{m} \exp \left[-\frac{d \lambda}{p^{1 /(d-1)}}\right]
$$

Now we are ready to prove our lower bound on $P(B, d, \ell, 2, p)$. Let $\mathcal{S}$ denote the set of sequences $\left(a_{i}, \mathbf{b}_{i}\right)_{i=1}^{m}$ that satisfy (5.11) and (5.12) and recall from Lemma 5.8 that

$$
|\mathcal{S}| \geq\left(\frac{8}{\gamma p}\right)^{m}
$$

It then follows from Lemma 5.7(ii), (iii) and from (5.16) that

$$
P(B, d, \ell, 2, p) \geq \sum_{\left(a_{i}, \mathbf{b}_{i}\right)_{i=1}^{m} \in \mathcal{S}} \mathbb{P}\left(\mathcal{G}\left(\left(a_{i}, \mathbf{b}_{i}\right)_{i=1}^{m}\right)\right) \geq p^{2 d+1} 2^{m} \exp \left[-\frac{d \lambda}{p^{1 /(d-1)}}\right] .
$$

Recall from Lemma 5.8 that $m=\gamma p^{-1 /(2 d-2)}$. Since $\log (1 / p) \ll p^{-1 /(2 d-2)}$ for $p$ sufficiently small, it follows that

$$
\begin{aligned}
P(B, d, \ell, 2, p) & \geq p^{2 d+1} \exp \left[\frac{\gamma \log 8}{p^{1 /(2 d-2)}}-\frac{d \lambda}{p^{1 /(d-1)}}\right] \\
& \geq \exp \left[\frac{2 \gamma}{p^{1 /(2 d-2)}}-\frac{d \lambda}{p^{1 /(d-1)}}\right]
\end{aligned}
$$

as claimed. 
Now we will show that the right-hand side of (5.13) is large enough that it is very likely that some fairly large cube in $[n]^{d} \times[2]^{\ell}$ is internally semispanned. In particular, the $2 \gamma p^{-1 /(2 d-2)}$ term in the exponent on the righthand side of (5.13) will allow us to prove Lemma 5.1.

Proof of Lemma 5.1. Recall that we want to show that if $c$ satisfies (5.1) and $p$ satisfies (5.2), then $P(n, d, \ell, 2, p) \rightarrow 1$ as $n \rightarrow \infty$. A standard coupling argument shows that $P(n, d, \ell, 2, p)$ is increasing in $p$, so it is enough to prove the lemma under the assumption that

$$
p \leq\left(\frac{\lambda(d+\ell, \ell+2)^{2}}{d^{2} \log n}\right)^{d-1} .
$$

Let $B=p^{-3 /(d-1)}$ and partition $[n]^{d} \times[2]^{\ell}$ into cubes of the form $[B]^{d} \times[2]^{\ell}$. We want to show that with high probability at least one of these cubes is internally semi-spanned. To do this, we use the following claim, whose proof we postpone to the Appendix.

Claim 5.11. Let $d \geq 2$, let $\ell \geq 0$, and let $\gamma=\gamma(d, \ell)$ be as in (4.3). If c satisfies (5.1) and $p$ satisfies (5.2) and (5.17), then there exists a constant $\alpha>0$ such that

$$
\frac{2 \gamma}{p^{1 /(2 d-2)}}-\frac{d \lambda(d+\ell, \ell+2)}{p^{1 /(d-1)}} \geq \alpha(\log n)^{1 / 2}-d \log n
$$

for $n$ sufficiently large.

Let $I_{B}$ denote the event that at least one cube of the form $[B]^{d} \times[2]^{\ell}$ is internally semi-spanned and let $\lambda=\lambda(d+\ell, \ell+2)$. By Lemma 5.10, Claim 5.11, and the fact that $e^{(\log n)^{1 / 3}} \gg B$, we have

$$
\begin{aligned}
\mathbb{P}\left(I_{B}\right) & \geq 1-\left(1-\exp \left[\frac{2 \gamma}{p^{1 /(2 d-2)}}-\frac{d \lambda}{p^{1 /(d-1)}}\right]\right)^{(n / B)^{d}} \\
& \geq 1-\exp \left[-\left(\frac{n}{B}\right)^{d} \exp \left(\frac{2 \gamma}{p^{1 /(2 d-2)}}-\frac{d \lambda}{p^{1 /(d-1)}}\right)\right] \\
& \geq 1-\exp \left[-\left(\frac{n}{B}\right)^{d} \exp \left(\alpha(\log n)^{1 / 2}-d \log n\right)\right] \\
& \geq 1-\exp \left[-\exp \left(\alpha(\log n)^{1 / 2}-(\log n)^{1 / 3}\right)\right] \\
& =1-o(1) .
\end{aligned}
$$

It is easy to see that if a cube of the form $[B]^{d} \times[2]^{\ell}$ is internally semispanned and every cuboid of the form $[B]^{t-1} \times\{1\} \times[B]^{d-t} \times \mathbf{1}^{\ell}$ is occupied, then the initially infected set $A$ semi-percolates in $C^{*}\left([n]^{d} \times[2]^{\ell}, 2\right)$. By (5.17), for all $d \geq 2$, we have $p \ll(\log n)^{-1 / 2}$. So, by the definition of $B$, the probability that some cuboid of the form $[B]^{t-1} \times\{1\} \times[B]^{d-t} \times \mathbf{1}^{\ell}$ is unoccupied is at most

$$
d n^{d}(1-p)^{B^{d-1}} \leq d n^{d} e^{-p^{-2}}=o(1) .
$$


Finally, because the events "a cube of the form $[B]^{d} \times[2]^{\ell}$ is internally semispanned" and "a cuboid of the form $[B]^{t-1} \times\{1\} \times[B]^{d-t} \times \mathbf{1}^{\ell}$ is occupied" are increasing, Harris's Lemma implies that $P(n, d, \ell, 2, p) \rightarrow 1$ as $n \rightarrow \infty$.

\section{Proofs of Theorems 1.4 And 4.1}

In this section, we complete the proof of Theorem 4.1 and use it to deduce Theorem 1.4. Note that Lemma 5.1 proves Theorem 4.1 for $r=2$, all $d \geq 2$, and all $\ell \geq 0$. The rest of the proof of Theorem 4.1 is an inductive argument that is very similar to the one used in [5]. However, we face a number of technical complications not present in [5]. So, in spite of the many similarities, we will give almost all of the details of the proof.

Recall that we wish to show that for all $d \geq r$ and all $\ell \geq 0$, if $p$ satisfies (4.7), then $P(n, d, \ell, r, p) \rightarrow 1$ as $n \rightarrow \infty$. We will assume that Theorem 4.1 holds for all $r^{\prime}<r$, all $d \geq r^{\prime}$, and all $\ell \geq 0$. In order to carry out the induction, we need two lemmas. The first lemma is due to Holroyd [22, Lemma 2]. We will need it for the case $r=3$.

Lemma 6.1. For any $d \geq 3, \ell \geq 0$, and $\varepsilon>0$, if $n$ is sufficiently large and $p^{-2 d} \leq n^{\varepsilon}$, then

$$
P(n, d, \ell, 3, p) \geq \exp \left(-n^{1+\varepsilon}\right) .
$$

The second is due to Balogh, Bollobás, and Morris [5, Lemma 12].

Lemma 6.2. For each $d \geq r \geq 2$ and each $\ell \geq 0$, there exists a constant $\eta=$ $\eta(d, \ell, r)>0$ such that the following holds. Let $\varepsilon, p>0$, let $n, m \in \mathbb{N}$, and let $A \sim \operatorname{Bin}\left([n]^{d} \times[2]^{\ell}, p\right)$. If

$$
P(m, d-i, \ell+i, r-i, p) \geq 1-\eta \quad \text { for all } i \in[r-2]
$$

and if $M \leq n$ is such that $M / m$ is sufficiently large (depending on $d, \ell, r$, and $\varepsilon)$, then

$$
\mathbb{P}\left([n]^{d} \times \mathbf{1}^{\ell} \subseteq\left[A \cup\left([M]^{d} \times \mathbf{1}^{\ell}\right)\right]\right) \geq 1-\varepsilon ;
$$

in particular,

$$
P(n, d, \ell, r, p) \geq(1-\varepsilon) P(M, d, \ell, r, p) .
$$

Remark 6.3. Lemma 6.2 simply provides a lower bound on the the probability that the infected set grows from a smaller cuboid to a larger one. However, the role of $m$ in the statement of the lemma deserves some explanation. Let $t \geq m$ and suppose that $[t]^{d} \times \mathbf{1}^{\ell}$ is internally spanned. It follows from an observation in [2] that if (6.1) holds, then the probability that $[t+1]^{d} \times \mathbf{1}^{\ell}$ is not internally spanned is exponentially small in $t / m$. (For a proof of this statement, see, e.g., [5, Lemma 11].) So, Harris's Lemma and the assumption on $M / m$ imply that there exists $C>0$ such that

$$
\mathbb{P}\left([n]^{d} \times \mathbf{1}^{\ell} \subseteq\left[A \cup\left([M]^{d} \times \mathbf{1}^{\ell}\right)\right]\right) \geq \prod_{t=M}^{n-1}\left(1-C e^{-t / m}\right) \geq 1-\varepsilon,
$$

which is exactly (6.2). 
To prove Theorem 4.1, we will need to define quantities $m, M$, and $N$ such that $1 \ll m \ll M \ll N \ll n$. We will bound from below the probability of filling a cuboid of side length $M$. Then, using our induction hypothesis and Lemma 6.2, we will bound the probability that this cuboid grows to fill a cuboid of side length $N:=(\log n)^{3}$. Once we have bounded $P(N, d, \ell, r, p)$, it will be easy to show that, with high probability, there exists a copy of $[N]^{d} \times$ $\mathbf{1}^{\ell}$ in $[n]^{d} \times \mathbf{1}^{\ell}$ that is internally spanned and that, with high probability, this copy of $[N]^{d} \times \mathbf{1}^{\ell}$ grows to fill all of $[n]^{d} \times \mathbf{1}^{\ell}$.

In order to apply Lemma 6.2 in the proof of Theorem 4.1, we must take some care in choosing the values of $m$ and $M$. Recall that we want to define $m$ such that for all $i \in[r-2], P(m, d-i, \ell+i, r-i, p)$ is sufficiently close to 1 . If $m$ is such that

$$
p \geq\left(\frac{\lambda(d+\ell, \ell+r)}{\log _{(r-i-1)}(m)}-\frac{c_{d-i, \ell+i, r-i}}{\left(\log _{(r-i-1)}(m)\right)^{3 / 2}}\right)^{d-r+1},
$$

then the desired lower bound on $P(m, d-i, \ell+i, r-i, p)$ follows from the induction hypothesis. Comparing (6.3) to the bound on $p$ in (4.7) suggests that it is reasonable to define $m$ such that $\log _{(r-2)}(m)$ is close to $\log _{(r-1)}(n)$. Recall also from Lemma 6.2 that we want to define $M$ such that $M / m \rightarrow$ $\infty$ as $n \rightarrow \infty$. Furthermore, it will turn out that we want $\log _{(r-2)}(M)$ to be slightly less than $\log _{(r-1)}(n)$. However, how close $\log _{(r-2)}(m)$ and $\log _{(r-2)}(M)$ must be to $\log _{(r-1)}(n)$ depends on $n$, which complicates the argument slightly.

First, let

$$
N=(\log n)^{3} .
$$

Given $d, \ell$, and $r$, let $\lambda=\lambda(d+\ell, \ell+r)$ be as in (1.3). We define $M, m$, and a third quantity $\delta$ such that $M$ is the largest positive value such that

$$
\begin{aligned}
\delta & =\frac{2^{-r} \gamma(d-r+2, \ell+r-2)}{\lambda}\left(\log _{(r-2)}(M)\right)^{-1 / 2}, \\
\log _{(r-2)}(M) & =(1-\delta) \log _{(r-1)}(n),
\end{aligned}
$$

and

$$
\log _{(r-2)}(m)=(1-2 \delta) \log _{(r-1)}(n) .
$$

It is not necessarily obvious from (6.4)-(6.6) that $M$ and $m$ are welldefined. To see that these quantities are indeed well-defined for $n$ sufficiently large, let $c=2^{-r} \gamma(d-r+2, \ell+r-2) / \lambda$ and observe that by (6.4), we may rewrite (6.5) as

$$
\log _{(r-2)}(M)=\left(1-c\left(\log _{(r-2)}(M)\right)^{-1 / 2}\right) \log _{(r-1)}(n) .
$$

Now let $y=\log _{(r-1)}(n)$ and let

$$
f(x)=x-\left(1-c x^{-1 / 2}\right) y .
$$


Elementary calculations show that for $n$ sufficiently large, $f$ has at least one and at most two positive real roots, at least one of which is larger than 1 . Let $x_{0}$ be the larger (or only) positive real root of $f$. Then we may define $M$ by $\log _{(r-2)}(M)=x_{0}$, which is exactly (6.7).

We note that $\delta \rightarrow 0$ as $n \rightarrow \infty$. (This convergence to 0 , which we have not been able to avoid, is the source of most of the technical complications in the proof of Theorem 4.1.) Also, observe that (6.4) and (6.5) imply that there exists a constant $C>0$ such that

$$
\delta \geq C\left(\log _{(r-1)}(n)\right)^{-1 / 2}
$$

for $n$ sufficiently large.

We are now ready to proceed with the proof of Theorem 4.1.

Proof of Theorem 4.1. As stated above, Lemma 5.1 gives the result for $r=2$. So, suppose that $r \geq 3$ and that for all $r^{\prime}<r$, the result holds for all $d \geq r^{\prime}$ and for all $\ell \geq 0$.

We begin by proving a lower bound on $P(M, d, \ell, r, p)$.

Claim 6.4. We have $P(M, d, \ell, r, p) \geq 1 / n$ as $n \rightarrow \infty$.

Proof. To prove the claim for $r=3$, we first observe that

$$
p^{-2 d} \leq(\log \log n)^{4 d^{2}} \leq M^{\delta} .
$$

The first inequality follows from (4.7). To see the second inequality, note that by (6.4) and (6.8),

$$
\delta \log M \geq C^{\prime}(\log \log n)^{1 / 2} \gg 4 d^{2} \log \log \log n .
$$

Then, by Lemma 6.1 and (6.5),

$$
P(M, d, \ell, 3, p) \geq \exp \left(-M^{1+\delta}\right)=\exp \left(-(\log n)^{1-\delta^{2}}\right) \geq 1 / n
$$

for $n$ sufficiently large.

To prove the claim for $r \geq 4$, it suffices to bound $P(M, d, \ell, r, p)$ from below by the probability that $[M]^{d} \times \mathbf{1}^{\ell}$ is full. To do this, we first show that

$$
\log M \ll(1-\delta) \log \log n .
$$

Observe that for all $k \geq 2$,

$$
\left(\log _{(k)}(n)\right)^{1-\delta} \ll(1-\delta) \log _{(k)}(n) .
$$

(To see that (6.10) indeed holds for $n$ sufficiently large, take logarithms and note that $\log (1-\delta) \geq-2 \delta$ for $\delta$ sufficiently small.) If we iteratively exponentiate both sides of (6.5) and apply (6.10), we see that for all $i \leq r-3$,

$$
\log _{(r-2-i)}(M) \leq\left(\log _{(r-1-i)}(n)\right)^{1-\delta} \ll(1-\delta) \log _{(r-1-i)}(n) .
$$

This yields (6.9).

We then observe that, by (6.9),

$$
P(M, d, \ell, r, p) \geq p^{M^{d}} \geq \exp \left(-\log (1 / p)(\log n)^{1-\delta}\right),
$$


which means that we are done if we can show that

$$
\exp \left(-\log (1 / p)(\log n)^{1-\delta}\right) \gg \frac{1}{n}
$$

If we take logarithms twice in (6.11), we see that it is enough to show that $\log \log (1 / p) \ll \delta \log \log n$. Observe that (6.8) and the fact that $1 / p \leq$ $c^{\prime} \log _{(r-1)}(n)$ imply that for all $r \geq 4$, we have

$$
\delta \log \log n \geq C^{\prime} \frac{\log \log n}{\left(\log _{(r-1)}(n)\right)^{1 / 2}} \gg \log _{(r+1)}(n) \geq \log \log (1 / p)
$$

as required to prove (6.11). This proves the claim.

Now we wish to use Lemma 6.2 to show that $P(N, d, \ell, r, p) \geq 1 / 2 n$ for all $n$ sufficiently large.

First, we claim that $M / m \rightarrow \infty$ as $n \rightarrow \infty$. For $r \geq 4$, this is easy to see. For $r=3$, we observe that by (6.5), (6.6), and (6.8),

$$
\frac{M}{m}=(\log n)^{\delta}=\exp (\delta \log \log n) \geq \exp \left(C^{\prime}(\log \log n)^{1 / 2}\right),
$$

which tends to infinity as $n \rightarrow \infty$.

Next, we show that our induction hypothesis implies that (6.1) holds, i.e., that $A$ is likely to semi-percolate in the lower-threshold sets adjacent to $[m]^{d} \times[2]^{\ell}$. Once we have done so, we will be ready to apply Lemma 6.2.

Claim 6.5. For all $i \in[r-2], P(m, d-i, \ell+i, r-i, p) \rightarrow 1$ as $n \rightarrow \infty$.

Proof. Let $\lambda=\lambda(d+\ell, \ell+r)$. By induction, it is enough to show that for all $i \in[r-2]$, with $c_{d, \ell, r}$ as in (4.5), we have

$$
\begin{aligned}
p & \geq\left(\frac{\lambda(1-2 \delta)}{\log _{(r-2)}(m)}-\frac{c_{d, \ell, r}(1-2 \delta)^{3 / 2}}{\left(\log _{(r-2)}(m)\right)^{3 / 2}}\right)^{d-r+1} \\
& \geq\left(\frac{\lambda}{\log _{(r-i-1)}(m)}-\frac{c_{d-i, \ell+i, r-i}}{\left(\log _{(r-i-1)}(m)\right)^{3 / 2}}\right)^{d-r+1},
\end{aligned}
$$

where the first inequality follows from (4.7) and (6.6). For $i \geq 2$, the second inequality is easy to see. For $i=1$, we need to show that

$$
\frac{\lambda(1-2 \delta)}{\log _{(r-2)}(m)}-\frac{c_{d, \ell, r}(1-2 \delta)^{3 / 2}}{\left(\log _{(r-2)}(m)\right)^{3 / 2}} \geq \frac{\lambda}{\log _{(r-2)}(m)}-\frac{c_{d-1, \ell+1, r-1}}{\left(\log _{(r-2)}(m)\right)^{3 / 2}} .
$$

Because $(1-2 \delta)^{3 / 2}<1$, it is enough to show that

$$
2 \delta \lambda\left(\log _{(r-2)}(m)\right)^{1 / 2}+c_{d, \ell, r} \leq c_{d-1, \ell+1, r-1} .
$$

Indeed, (6.4), the fact that $m \leq M$, and (4.6) imply that $2 \delta \lambda\left(\log _{(r-2)}(m)\right)^{1 / 2}+c_{d, \ell, r} \leq 2^{-r+1} \gamma(d-r+2, \ell+r-2)+c_{d, \ell, r}=c_{d-1, \ell+1, r-1}$.

It then follows from the induction hypothesis that for each $i \in[r-2]$, $P(m, d-i, \ell+i, r-i, p) \rightarrow 1$ as $n \rightarrow \infty$, as claimed. 
By Claim 6.5, we may apply Lemma 6.2 to $P(N, d, \ell, r, p)$. The lemma and Claim 6.4 imply that

$$
P(N, d, \ell, r, p) \geq(1-\varepsilon) P(M, d, \ell, r, p) \geq \frac{1}{2 n}
$$

for all $n$ sufficiently large. Since $(n / N)^{d} \gg 2 n$, with high probability, there exists a cuboid $K \times \mathbf{1}^{\ell} \subseteq[A]$ with $|K| \geq N^{d}$. So, by applying Lemma 6.2 again (this time with $N$ in place of $M$ ) and Harris's Lemma, we have

$$
P(n, d, \ell, r, p) \geq(1-o(1)) \mathbb{P}\left([n]^{d} \times \mathbf{1}^{\ell} \subseteq\left[A \cup\left(K \times \mathbf{1}^{\ell}\right)\right]\right)=1-o(1) .
$$

This completes the proof of Theorem 4.1.

The proof of Theorem 1.4 is immediate.

Proof of Theorem 1.4. Let $d \geq r \geq 2$ and let $n$ be sufficiently large. Let $c_{d, r}=c_{d, 0, r}$ and note that (4.5) implies that $c_{d, r}>0$. Applying Theorem 4.1 with $\ell=0$ shows that

$$
p_{c}\left([n]^{d}, r\right) \leq\left(\frac{\lambda(d, r)}{\log _{(r-1)}(n)}-\frac{c_{d, r}}{\left(\log _{(r-1)}(n)\right)^{3 / 2}}\right)^{d-r+1},
$$

as claimed.

\section{Open Questions}

It remains to improve the lower bound on the critical probability $p_{c}\left([n]^{d}, r\right)$ for values of $d \geq r \geq 2$ other than $d=r=2$. Given the difficulty of the proof of the lower bound in Theorem 1.3, this is likely to be much harder than the proof of Theorem 1.4, especially for $r \geq 3$. Note, however, that the upper bound on $p_{c}\left([n]^{2}, 2\right)$ in [17] gave the correct order of magnitude of the second term. Because the proof of Theorem 1.4 can be seen as a fairly natural generalization of the arguments in [17] to higher dimensions, we conjecture that it gives the correct order of magnitude of the second term in $p_{c}\left([n]^{d}, r\right)$ for all $d \geq r \geq 2$.

Conjecture 7.1. Let $d \geq r \geq 2$. As $n \rightarrow \infty$,

$$
p_{c}\left([n]^{d}, r\right)=\left(\frac{\lambda(d, r)}{\log _{(r-1)}(n)}-\Theta\left(\frac{1}{\left(\log _{(r-1)}(n)\right)^{3 / 2}}\right)\right)^{d-r+1} .
$$

\section{ACKNOWLEDGMents}

I am grateful to Rob Morris for pointing out that an earlier version of Claim 5.11 could be strengthened, which made it possible to strengthen the statement of Theorem 1.4. I also wish to thank Paul Balister, Richard Johnson, and Michał Przykucki for reading preliminary versions of the manuscript and for numerous helpful comments. Finally, I am grateful to the anonymous referees for many helpful comments that improved the presentation of the paper. 


\section{REFERENCES}

1. J. Adler and U. Lev, Bootstrap percolation: visualizations and applications, Braz. J. Phys. 33 (2003), no. 3, 641-644.

2. M. Aizenman and J. L. Lebowitz, Metastability effects in bootstrap percolation, J. Phys. A 21 (1988), no. 19, 3801-3813.

3. P. Balister, B. Bollobás, M. Przykucki, and P. Smith, Subcritical U-bootstrap percolation models have non-trivial phase transitions, Trans. Amer. Math. Soc. 368 (2016), no. $10,7385-7411$.

4. J. Balogh, B. Bollobás, H. Duminil-Copin, and R. Morris, The sharp threshold for bootstrap percolation in all dimensions, Trans. Amer. Math. Soc. 364 (2012), no. 5, 2667-2701.

5. J. Balogh, B. Bollobás, and R. Morris, Bootstrap percolation in three dimensions, Ann. Probab. 37 (2009), no. 4, 1329-1380.

6. J. Balogh and G. Pete, Random disease on the square grid, Random Structures Algorithms 134 (1998), no. 3-4, 409-422.

7. B. Bollobás, H. Duminil-Copin, R. Morris, and P. Smith, Universality of twodimensional critical cellular automata, Proc. London Math. Soc., to appear.

8. _ The sharp threshold for the Duarte model, Ann. Probab. 45 (2017), no. 6B, 4222-4272.

9. B. Bollobás, P. J. Smith, and A. J. Uzzell, Monotone cellular automata in a random environment, Combin. Probab. Comput. 24 (2015), no. 4, 687-722.

10. R. Cerf and E. N. M. Cirillo, Finite size scaling in three-dimensional bootstrap percolation, Ann. Probab. 27 (1999), no. 4, 1837-1850.

11. R. Cerf and F. Manzo, The threshold regime of finite volume bootstrap percolation, Stochastic Process. Appl. 101 (2002), no. 1, 69-82.

12. J. Chalupa, P. L. Leath, and G. R. Reich, Bootstrap percolation on a Bethe lattice, J. Phys. C 12 (1979), no. 1, L31-L35.

13. H. Duminil-Copin and A. E. Holroyd, Finite volume bootstrap percolation with threshold rules on $\mathbb{Z}^{2}$ : balanced case, preprint, http://www.ihes.fr/ duminil/, 2012.

14. H. Duminil-Copin and A. C. D. van Enter, Sharp metastability threshold for an anisotropic bootstrap percolation model, Ann. Probab. 41 (2013), no. 3A, 1218-1242.

15. H. Duminil-Copin, A. C. D. van Enter, and T. Hulshof, Higher order corrections for anisotropic bootstrap percolation, Probab. Theory Related Fields, to appear.

16. L. R. Fontes, R. H. Schonmann, and V. Sidoravicius, Stretched exponential fixation in stochastic Ising models at zero temperature, Comm. Math. Phys. 228 (2002), 495-518.

17. J. Gravner and A. E. Holroyd, Slow convergence in bootstrap percolation, Ann. Appl. Probab. 18 (2008), no. 3, 909-928.

18. J. Gravner, A. E. Holroyd, and R. Morris, A sharper threshold for bootstrap percolation in two dimensions, Probab. Theory Related Fields 153 (2012), no. 1, 1-23.

19. T. E. Harris, A lower bound for the critical probability in a certain percolation process, Math. Proc. Cambridge Philos. Soc. 26 (1960), 13-20.

20. I. Hartarsky and R. Morris, The second term for two-neighbour bootstrap percolation in two dimensions, in preparation.

21. A. E. Holroyd, Sharp metastability threshold for two-dimensional bootstrap percolation, Probab. Theory Related Fields 125 (2003), no. 2, 195-224.

22. - The metastability threshold for modified bootstrap percolation in d dimensions, Electron. J. Probab. 11 (2006), 418-433.

23. R. Morris, Zero-temperature Glauber dynamics on $\mathbb{Z}^{d}$, Probab. Theory Related Fields 149 (2011), no. 3, 417-434.

24. R. H. Schonmann, On the behavior of some cellular automata related to bootstrap percolation, Ann. Probab. 20 (1992), no. 1, 174-193. 
25. A. C. D. van Enter, Proof of Straley's argument for bootstrap percolation, J. Stat. Phys. 48 (1987), 943-945.

26. A. C. D. van Enter and A. Fey, Metastability threshold for anisotropic bootstrap percolation in three dimensions, J. Stat. Phys. 147 (2012), no. 1, 97-112.

\section{APPENDIX A.}

Here we give the proofs of results from the paper that, while straightforward, rely on somewhat lengthy calculations.

Proof of Proposition 3.1. Recall that we wish to bound $\left|g_{k}^{\prime}(z)\right|$ from above for all $k \geq 1$ and all $z \geq 1$. By (1.2),

$$
\left|g_{k}^{\prime}(z)\right|=\left|\frac{e^{-z} \beta_{k}^{\prime}\left(1-e^{-z}\right)}{\beta_{k}\left(1-e^{-z}\right)}\right| .
$$

We begin by bounding $\beta_{k}^{\prime}\left(1-e^{-z}\right)$ from above. (Recall that $\beta_{k}$ is increasing on $(0,1)$.) First, differentiating both sides of $(3.3)$ gives

$2 \beta_{k}(u) \beta_{k}^{\prime}(u)=k(1-u)^{k-1} \beta_{k}(u)+\left(1-(1-u)^{k}\right) \beta_{k}^{\prime}(u)+(1-u)^{k}-k u(1-u)^{k-1}$.

We may rewrite this as

$$
\beta_{k}^{\prime}(u)=\frac{k(1-u)^{k-1} \beta_{k}(u)+(1-u)^{k}-k u(1-u)^{k-1}}{2 \beta_{k}(u)-1+(1-u)^{k}} .
$$

It follows from (1.1) and the fact that $\beta_{k}(u)<1$ for all $u \in(0,1)$ that

$$
\begin{aligned}
\beta_{k}^{\prime}(u) & =\frac{k(1-u)^{k-1} \beta_{k}(u)+(1-u)^{k}-k u(1-u)^{k-1}}{\sqrt{1+(4 u-2)(1-u)^{k}+(1-u)^{2 k}}} \\
& \leq \frac{k(1-u)^{k-1}+(1-u)^{k}-k u(1-u)^{k-1}}{\sqrt{1+(4 u-2)(1-u)^{k}+(1-u)^{2 k}}} \\
& =\frac{(k+1)(1-u)^{k}}{\sqrt{1+(4 u-2)(1-u)^{k}+(1-u)^{2 k}}} .
\end{aligned}
$$

Observe that the denominator of the right-hand side of (A.2) is at least 1 for all $u \geq 1 / 2$. If $z \geq 1$, then $1-e^{-z} \geq 1 / 2$, so for all such $z$, we have

$$
\beta_{k}^{\prime}\left(1-e^{-z}\right) \leq(k+1) e^{-z k} .
$$

Next, observe that for $u \geq 0$, the quantity under the square root on the right-hand side of $(1.1)$ is at least $\left(1-(1-u)^{k}\right)^{2}$, which means that

$$
\beta_{k}(u) \geq 1-(1-u)^{k}
$$

for all $u \in(0,1)$.

When we combine (A.3) and (A.4) with (A.1), we find that

$$
\left|g_{k}^{\prime}(z)\right| \leq \frac{(k+1) e^{-z(k+1)}}{1-e^{-z k}}=\frac{k+1}{e^{z(k+1)}-e^{z}} \leq \frac{2}{e^{2}-2}<\frac{1}{2},
$$

which is what we wanted. 
Proof of Claim 5.11. The claim is a lower bound on $\log (P(B, d, \ell, 2, p))$. Recall that $c$ is the constant from Lemma 5.1 and that

$$
\left(\frac{\lambda(d+\ell, \ell+2)}{\log n}-\frac{c}{(\log n)^{3 / 2}}\right)^{d-1} \leq p \leq\left(\frac{\lambda(d+\ell, \ell+2)^{2}}{d^{2} \log n}\right)^{d-1},
$$

where the upper bound is the assumption (5.17).

Let $\lambda=\lambda(d+\ell, \ell+2)$. By (A.5),

$$
\frac{2 \gamma}{p^{1 /(2 d-2)}}-\frac{d \lambda}{p^{1 /(d-1)}} \geq 2 \gamma\left(\frac{\lambda^{2}}{d^{2} \log n}\right)^{-1 / 2}-d \log n\left(1-\frac{c}{\lambda(\log n)^{1 / 2}}\right)^{-1} .
$$

Let $\varepsilon>0$ be sufficiently small. If $x$ is sufficiently small, then $(1-x)^{-1} \leq$ $1+(1+\varepsilon) x$. Hence, for $n$ sufficiently large, we have

$$
\frac{2 \gamma}{p^{1 /(2 d-2)}}-\frac{d \lambda}{p^{1 /(d-1)}} \geq d \log n \cdot \frac{2 \gamma}{\lambda(\log n)^{1 / 2}}-d \log n\left(1+\frac{(1+\varepsilon) c}{\lambda(\log n)^{1 / 2}}\right) .
$$

By (5.1),

$$
(1+\varepsilon) c<\frac{4 c}{3}<2 \gamma
$$

It follows that there exists $\alpha>0$ such that the right-hand side of (A.6) is at least $\alpha(\log n)^{1 / 2}-d \log n$, which is what we wanted.

Department of Mathematics and Statistics, Grinnell College, Grinnell, IA 50112, USA

E-mail address: uzzellan@grinnell.edu 